\title{
Régime thermique des sols et rôle du gel dans la dynamique des versants d'un milieu subéquatorial d'altitude : les Andes centrales du Pérou
}

\author{
Temperature Fluctuations in Soil and Frost Action on the \\ Dynamics of Slopes in a Subequatorial Mountain Environment; \\ the Central Andes of Peru \\ Regimen térmico de los suelos y acción del hielo sobre la \\ dinámica de las vertientes en el ambiente de la alta montaña \\ subecuatorial: los Andes Centrales del Perù
}

\section{Bernard Francou}

Volume 43, numéro 1, 1989

URI : https://id.erudit.org/iderudit/032757ar

DOI : https://doi.org/10.7202/032757ar

\section{Aller au sommaire du numéro}

\section{Éditeur(s)}

Les Presses de l'Université de Montréal

\section{ISSN}

0705-7199 (imprimé)

1492-143X (numérique)

\section{Découvrir la revue}

\section{Citer cet article}

Francou, B. (1989). Régime thermique des sols et rôle du gel dans la dynamique des versants d'un milieu subéquatorial d'altitude : les Andes centrales du Pérou. Géographie physique et Quaternaire, 43(1), 97-112.

https://doi.org/10.7202/032757ar

\section{Résumé de l'article}

Le gel et son action sont étudiés ici dans le milieu de la haute montagne subéquatoriale. La fréquence du gel est journalière et son intensité mesurée sous abri à $5000 \mathrm{~m}$ ne descend guère au-delà de $-6^{\circ} \mathrm{C}$. Dans le sol, les températures relevées à $10 \mathrm{~cm}$ ne dépassent pas $-2^{\circ} \mathrm{C}$ et le gel est atteint moins de 50 jours par an. L'épaisseur de la couche du sol parcourue par le front de gel est limitée à 10-15 cm. Le gel pénètre mieux dans les sols fins (sables silteux) et bien drainés. Dans les parois rocheuses, le gel est " pelliculaire » et limité à quelques heures jusqu'à $5200 \mathrm{~m}$. Dans les conditions actuelles, la formation d'un pergélisol dans le sol ou dans la roche n'est pas possible en dessous de 5 $200 \mathrm{~m}$. En première hypothèse, on peut mettre en relation les caractéristiques du gel ainsi définies avec la faible efficacité de la gélifraction, si l'on met à part quelques faciès plus gélifs qui fournissent des éléments de petite taille. Dans le sol, à côté de l'action de la glace d'exsudation, on met en évidence le rôle de la cryoreptation dans le déplacement des particules sur les versants. En plus des microformes de tri très répandues dans l'élaboration desquelles les mécanismes cryo-induits et le ruissellement de lavage se combinent étroitement, on insiste sur les particularités que revêtent les éboulis et les lobes à front pierreux dans ce milieu. La principale originalité consiste en la mise en place de dépôts stratifiés. Dans une perspective paléomorphologique, la présence de certaines formes inactives révèle que le cycle de gel-dégel n'a pas toujours eu les mêmes tendances depuis la fin du dernier pléniglaciaire et que les conditions ont dû être réunies dans le passé pour permettre des gels plus intenses et plus durables. 


\title{
RÉGIME THERMIQUE DES SOLS ET RÔLE DU GEL DANS LA DYNAMIQUE DES VERSANTS D'UN MILIEU SUBÉQUATORIAL D'ALTITUDE: LES ANDES CENTRALES DU PÉROU
}

\author{
Bernard FRANCOU, Centre de géomorphologie du CNRS, Rue des Tilleuls, 14000 Caen, France.
}

RÉSUMÉ Le gel et son action sont étudiés ici dans le milieu de la haute montagne subéquatoriale. La fréquence du gel est journalière et son intensité mesurée sous abri à $5000 \mathrm{~m}$ ne descend guère au-delà de $-6^{\circ} \mathrm{C}$. Dans le sol, les températures relevées à $10 \mathrm{~cm}$ ne dépassent pas $-2^{\circ} \mathrm{C}$ et le gel est atteint moins de 50 jours par an. L'épaisseur de la couche du sol parcourue par le front de gel est limitée à 10-15 cm. Le gel pénètre mieux dans les sols fins (sables silteux) et bien drainés. Dans les parois rocheuses, le gel est "pelliculaire" et limité à quelques heures jusqu'à $5200 \mathrm{~m}$. Dans les conditions actuelles, la formation d'un pergélisol dans le sol ou dans la roche n'est pas possible en dessous de $5200 \mathrm{~m}$. En première hypothèse, on peut mettre en relation les caractéristiques du gel ainsi définies avec la faible efficacité de la gélifraction, si l'on met à part quelques faciès plus gélifs qui fournissent des éléments de petite taille. Dans le sol, à côté de l'action de la glace d'exsudation, on met en évidence le rôle de la cryoreptation dans le déplacement des particules sur les versants. En plus des microformes de tri très répandues dans l'élaboration desquelles les mécanismes cryoinduits et le ruissellement de lavage se combinent étroitement, on insiste sur les particularités que revêtent les éboulis et les lobes à front pierreux dans ce milieu. La principale originalité consiste en la mise en place de dépôts stratifiés. Dans une perspective paléomorphologique, la présence de certaines formes inactives révèle que le cycle de geldégel n'a pas toujours eu les mêmes tendances depuis la fin du dernier pléniglaciaire et que les conditions ont dû être réunies dans le passé pour permettre des gels plus intenses et plus durables.
ABSTRACT Temperature fluctuations in soil and frost action on the dynamics of slopes in a subequatorial mountain environment; the Central Andes of Peru. Frost and frost action are studied here in a subequatorial high mountain environment. Frost occurs every day but minimum temperatures under shelter at $5000 \mathrm{~m}$ generally do not exceed $-6^{\circ} \mathrm{C}$. In the ground at $10 \mathrm{~cm}$ depth, $-2^{\circ} \mathrm{C}$ is the minimum that was measured and depth of freezing is less than $10-15 \mathrm{~cm}$. Frost penetration is deeper in sandy and silty soils than in openwork gravels, except when they are saturated. In the rockfaces, frost is shallow and limited to a few hours. Present day conditions do not allow the formation of permafrost in the ground and in the rockfaces below $5200 \mathrm{~m}$. With such characteristics, and in spite of its frequency frost cannot presently trigger efficient rock splitting except in the very porous rocks which yield many small particles. In the ground surficial movement of particles is induced by needle ice but also by frost creep. Sorted patterned ground is very common on the slopes. Sorting is due to frost action and slope washing. Talus slope and stone banked lobes in this environment often are stratified deposits. In a paleoclimatic view analysis of inactive forms allows us to assume that frost cycle did not always take the same characteristics since the end of the last glaciation. In one or several periods severe climatic conditions caused more intensive and prolonged frosts.
RESUMEN Regimen térmico de los suelos y acción del hielo sobre la dinámica de las vertientes en el ambiente de la alta montaña subecuatorial: los Andes Centrales del Perù. Se estudia en este artículo el hielo y su acción en el ambiente de la alta montaña subecuatorial. La helada se produce cada día y su intensidad en el abrigo a $5000 \mathrm{~m}$ generalmente no sobrepasa $-6^{\circ} \mathrm{C}$. En el suelo, las temperaturas mínimas recogidas a $10 \mathrm{~cm}$ no rebasan $-2^{\circ} \mathrm{C}$ y el hielo se forma menos de 50 días en el ano. La profundidad alcanzada por el frente de hielo se limita a 10$15 \mathrm{~cm}$. El frío penetra mas los suelos con fracción arenosa-siltosa abundante y no muy húmedos. En las paredes recosas, hasta $5200 \mathrm{~m}$ de altura, la capa helada es muy delgada y limitada a unas horas. En las condiciones presentes, la formación de un permafrost en el suelo o en las rocas es imposible bajo $5200 \mathrm{~m}$. Se emite como hipótesis que este tipo de heladas puede explicar la poco eficacia de la cryoclastía, excepto sobre algunas rocas alteradas que producen actualmente muchos elementos de pequeño tamaño. En el suelo, se pone en evidencia, junto al acción de los pipkrakes, la importencia del frost creep en el desplazamiento de las partículas sobre las vertientes. Además de las microformas con selección de los materiales elaboradas por el acción conjunto del hielo y del escurrimiento, se destaca la originalidad de los talos y de los lóbulos de congelifluxión en este ambiente. El rasgo mas característico es la estratificación de los depósitos. De un punto de visto paleomorphológico, la presencia de formas inactivas demostra que el ciclo de congelamiento no tuvo siempre las mismas características desde el fin de la última glaciación. Durante uno o varios períodos, condiciones climaticas mas severas tuvieron que provocar heladas mas intensas y durables. 


\section{INTRODUCTION}

La zone étudiée concerne la Cordillère centrale située sous le $12^{\circ} \mathrm{S}$ et dominant le littoral désertique du Pérou à proximité de Lima (fig. 1). Dans une coupe N-S des Andes, elle correspond à un milieu subéquatorial d'altitude dans lequel l'amplitude saisonnière des températures est faible et où les précipitations sont assez importantes pour que le niveau des neiges permanentes et l'isotherme $0^{\circ} \mathrm{C}$ soient à peu près confondus (Troll, 1968; Schwerdtfeger, 1976). Comme on le voit sur la figure 2, cela implique un étage périglaciaire d'extension limitée, entre $4700 \mathrm{~m}$, niveau où prennent fin les steppes d'altitude (punas bravas) et $5100 \mathrm{~m}$ environ où se situe la ligne d'équilibre des glaciers.

Ce type de milieu a déjà fait l'objet de plusieurs études géomorphologiques consistant surtout en un repérage des formes attribuables à l'action du gel (Troll, 1944; Dollfus,

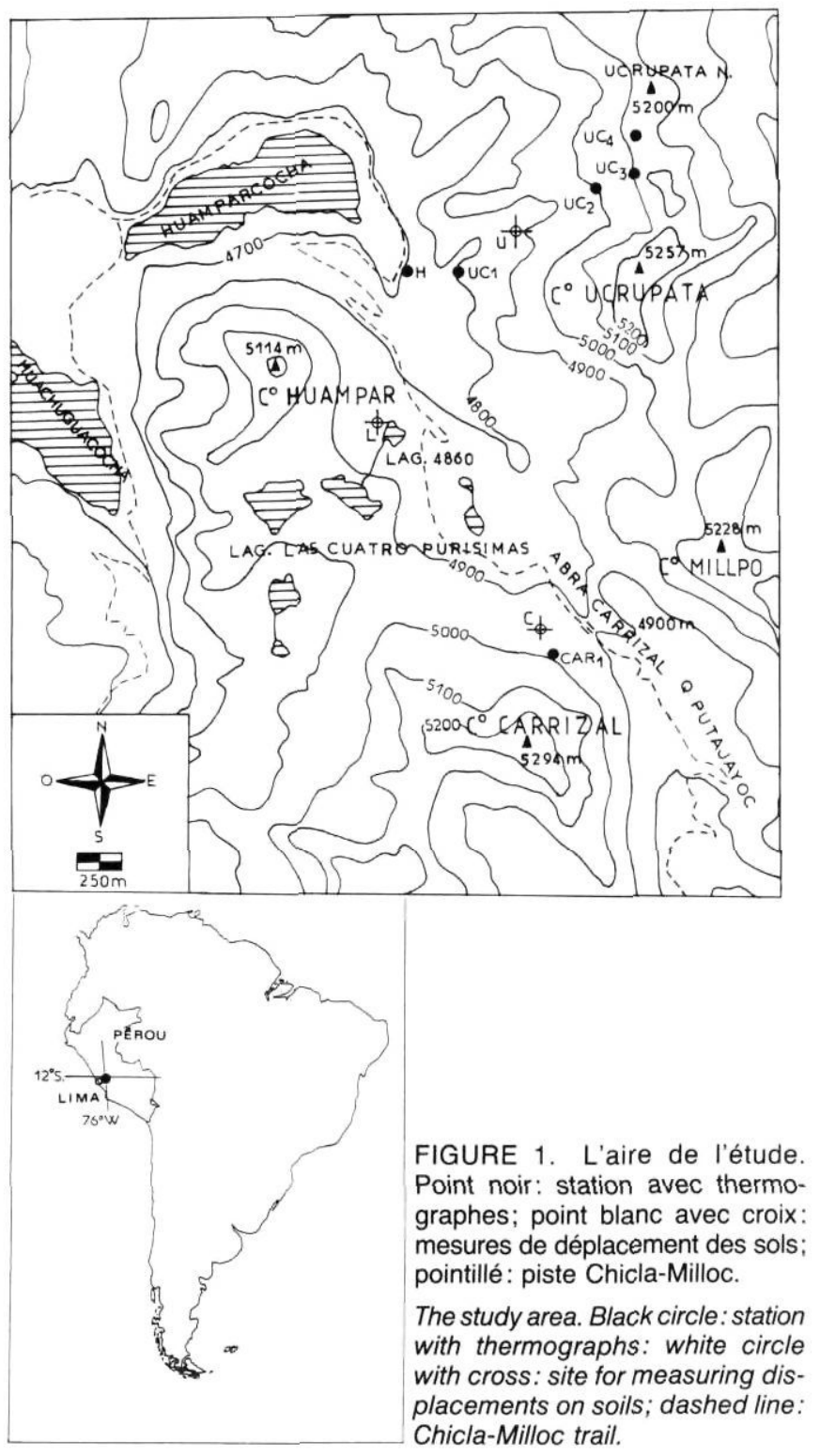

1965; Graf, 1971, 1976; Hastenrath, 1977). Mais des mesures plus poussées concernant les dynamiques de versant ont été conduites dans le milieu plus humide du páramo vénézuélien, avec mesures des températures et des déplacements de sols figurés, d'abord par Schubert (1979) puis plus récemment par Pérez (1984, 1985, 1986 et 1987). Toutes ces études ont insisté sur la fréquence du gel, imposée par son rythme journalier. Mais sa brièveté (une dizaine d'heures) limite sa pénétration à une frange très superficielle du sol et de la roche et son action s'exerce avant tout sous la forme d'une glace d'exsudation (Tricart et Cailleux, 1967; Washburn, 1979).

\section{La démarche de notre travail est double:}

1. II s'agit d'abord d'étudier le régime thermique actuel des hautes Andes vers $5000 \mathrm{~m}$, en décrivant les conditions dans lesquelles s'effectue la pénétration du gel dans les matériaux, sols et parois, et ceci à partir d'un dispositif de mesure mis en place et suivi pendant plusieurs années.

2. II s'agit ensuite d'identifier les principales actions du gel sur les versants à partir de formes dont on a étudié la dynamique et le fonctionnement.

L'objectif de cette étude est de vérifier si, comme l'ont admis les travaux antérieurs, l'action du gel se limite essentiellement dans un tel milieu à celle de la glace d'exsudation. II convient de voir également si toutes les formes cryo-induites présentes sur les versants, notamment celles qui sont inactives à présent, peuvent se développer dans le cadre de l'actuel cycle de gel-dégel journalier. Cette dernière question pose le problème de la constance des caractéristiques actuelles du gel sur toute la durée du Tardiglaciaire et de l'Holocène, soit sur une période de 14000 ans environ (Mercer et al., 1977).

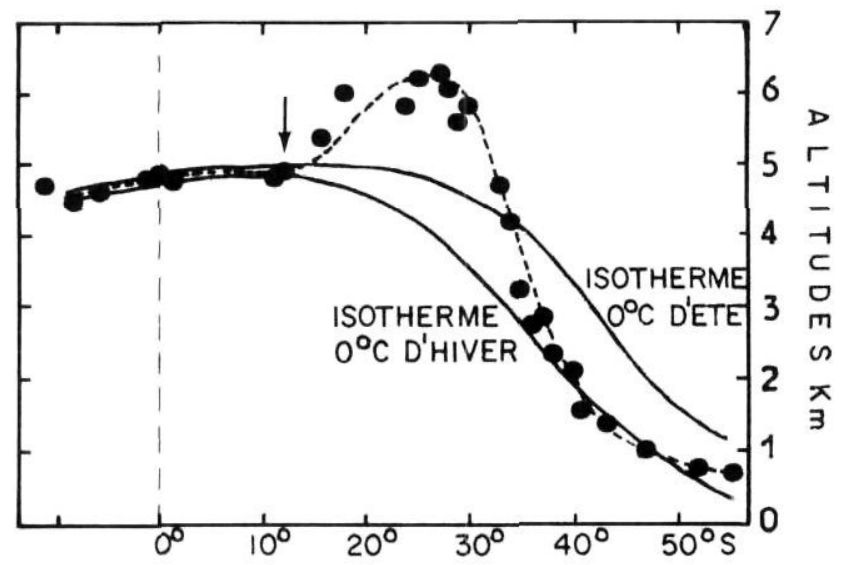

LATITUDE

FIGURE 2. Isothermes $0^{\circ} \mathrm{C}$ d'été et d'hiver et limites des neiges permanentes le long de la chaîne andine (points). La flèche indique la position de la chaîne étudiée (modifié d'après Schwerdtfeger, 1976).

Summer and winter limits of $0^{\circ} \mathrm{C}$ isotherms (solid line) and altitudes of the permament snowline (dashed line). Arrow shows position of the study area (modified after Schwerdtfeger, 1976). 


\section{LES OSCILLATIONS THERMIQUES AUTOUR DE $0^{\circ}$ C RELEVÉES DANS L'AIR ET DANS LE SOL AU-DESSUS DE $4000 \mathrm{M}$}

\section{I - LE RÉGIME THERMIQUE AU COURS DE L'ANNÉE DANS LES ANDES}

La série trentenaire recueillie à la station de Pachachaca à $4000 \mathrm{~m}$ d'altitude (fig. 1) a permis de définir en termes de probabilités le régime thermique annuel au niveau de l'étage de la puna (Francou, 1983). Ces calendriers de probabilités (Péguy, 1976) montrent une différence de comportement nette entre les maximums et les minimums moyens (fig. 3): les premiers n'oscillent guère au cours de l'année et leur médiane se maintient élevée, entre $14^{\circ} \mathrm{C}$ et $16^{\circ} \mathrm{C}$; les seconds décrivent au contraire un net mouvement saisonnier, isolant à cette altitude une période de 8 mois durant lesquels la température de gel a la meilleure chance d'être atteinte (d'avril à novembre) et dans ces 8 mois, les 3 mois secs où les gels les plus intenses peuvent avoir lieu (de juin à août). Dès $4000 \mathrm{~m}$, donc, le contraste thermique entre le jour et la nuit permet aux cycles de gel-dégel de se produire sur 8-9 mois de l'année.

\section{II - LA FRÉQUENCE ET LES TYPES DE TEMPÉRATURES NÉGATIVES RELEVÉES AUX STATIONS DE HUAMPAR SOUS ABRI ENTRE 4700 ET $5000 \mathrm{M}$}

On a installé à la fin de 1981 une série de stations thermographiques le long des deux versants dominant la Laguna Huampar (fig. 1). La figure 4 permet de localiser ces stations:

a) Le versant Ucrupata (fig. 4 et 5) est orienté SW et comporte entre 5000 et $5200 \mathrm{~m}$ un petit glacier de calotte et entre 4700 et $5000 \mathrm{~m}$ une série de dépôts morainiques abandonnés par le glacier depuis le maximum du Petit Âge glaciaire que l'on date dans la région de 1860 (Broggi, 1945); sur ces dépôts non végétalisés se développent une série de
MAXIMUMS - PACHACHACA

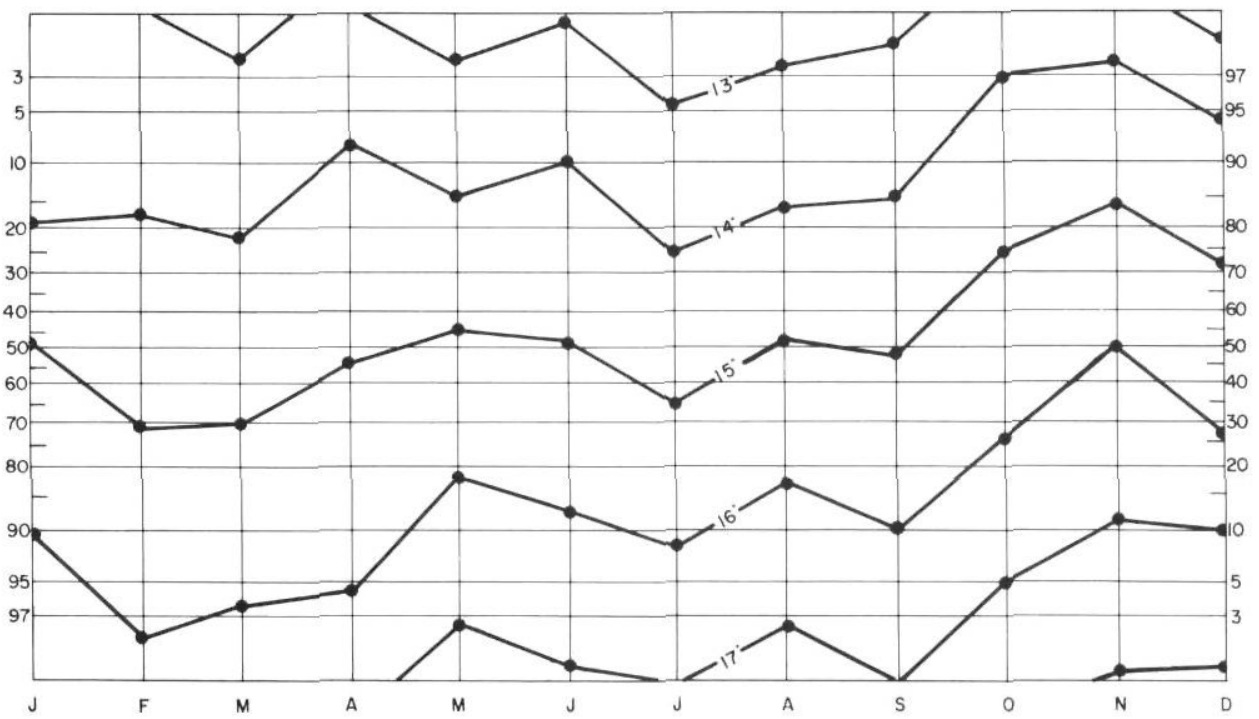

MINIMUMS - PACHACHACA

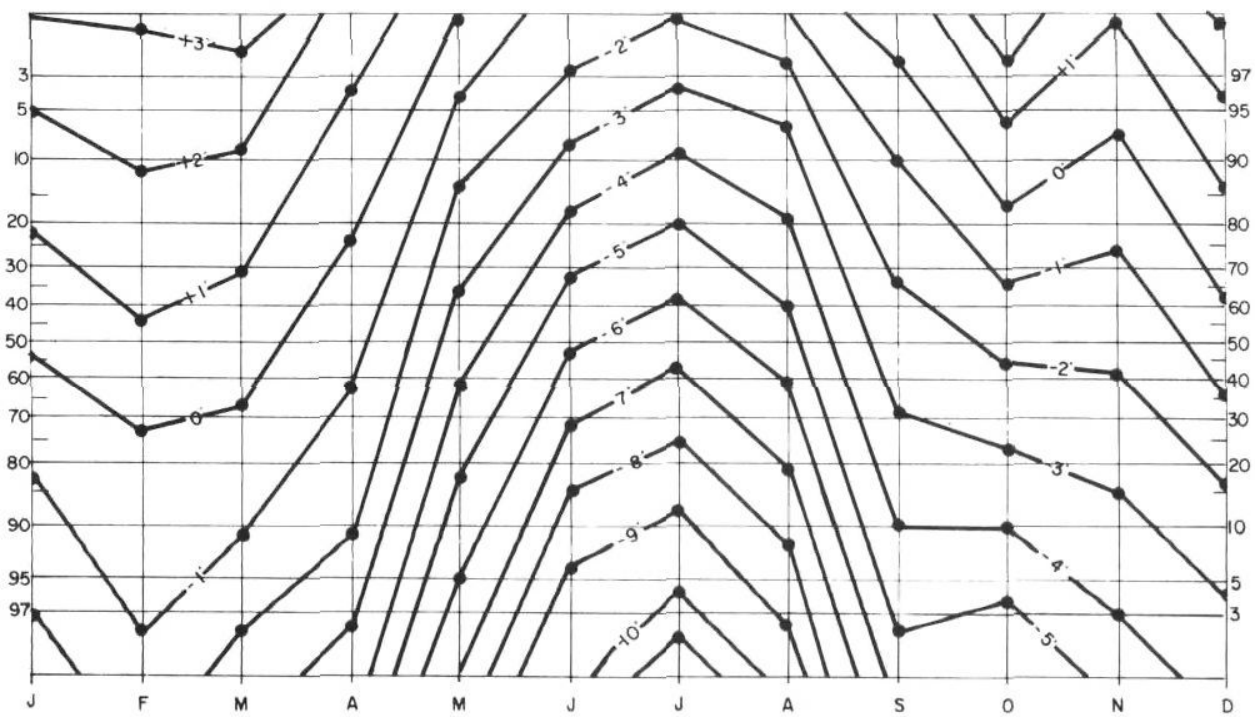

FIGURE 3. Calendriers des probabilités des maximums et minimums thermiques moyens à $\mathrm{Pa}$ chachaca $(4000 \mathrm{~m})$ à partir de la série 1949-1980. Pas et champ mensuels.

Probability calendars of maximum and minimum temperatures at $\mathrm{Pa}$ chachaca $(4000 \mathrm{~m})$ from 1949 to 1980. Period: one month. 


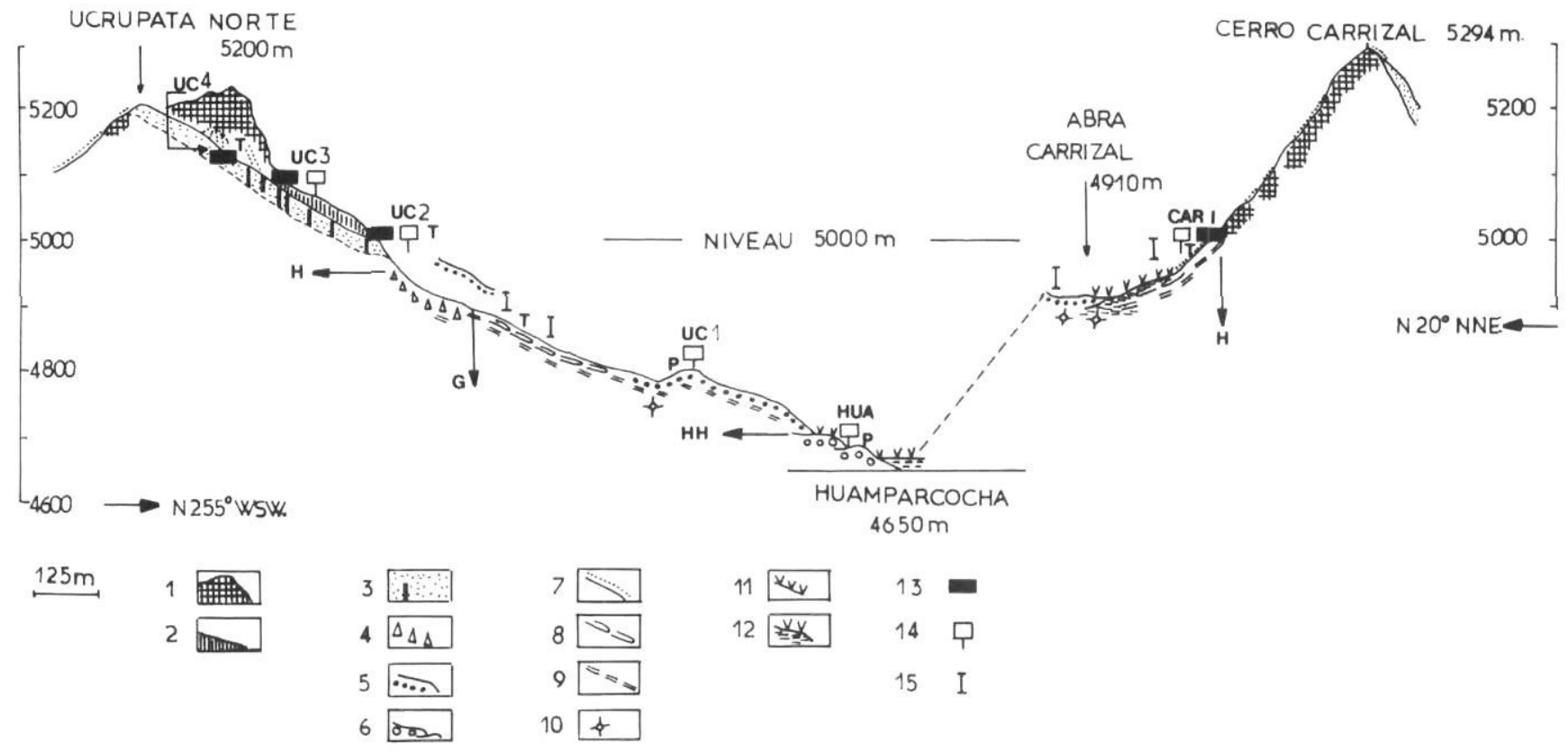

FIGURE 4. Les versants étudiés à Huampar et l'équipement mis en place. 1) Parois rocheuses; 2) roches moutonnées; 3) glacier; 4) moraines sub-actuelles; 5) moraines du Petit Âge glaciaire; 6) moraines anciennes; 7) talus d'éboulis actifs; 8 ) formes de la solifluxion; 9) sols striés; 10) sols à taches de terre; 11) steppe à Ichu; 12) sols hydromorphes avec Distichia muscoides; 13) thermosondes; 14) thermohydrographes sous abri; 15) balises alignées pour l'étude des déplacements sur les sols. T. Thermomètre à maxi-mini dans le sol; $\mathrm{H}$. limite supérieure des orophytes isolés; $\mathrm{HH}$. limite supérieure de la steppe à Ichu; G. limite du glacier en 1951; P. pluviomètre; $\$$ petit lac temporaire.
Studied slope at Huampar with the stations. 1) Rockfaces; 2) roches moutonnées; 3) glaciers; 4) very recent moraines; 5) Little lce Age moraines; 6) old moraines; 7) active talus slopes; 8) solifluction forms; 9) soil stripes; 10) nubbins; 11) Ichu steppe; 12) hydromorphic soils with Distichia muscoides; 13) temperature probes; 14) sheltered thermohydrographs; 15) marked lines for measuring displacements on soils; T. Maximini thermometers in ground; $H$. upper limit of isolated orophytes; HH. Upper limit of Ichu steppe; G. lower limit of glacier in 1951; P. Rain gauge; $\$$ ephemeral lagoon.

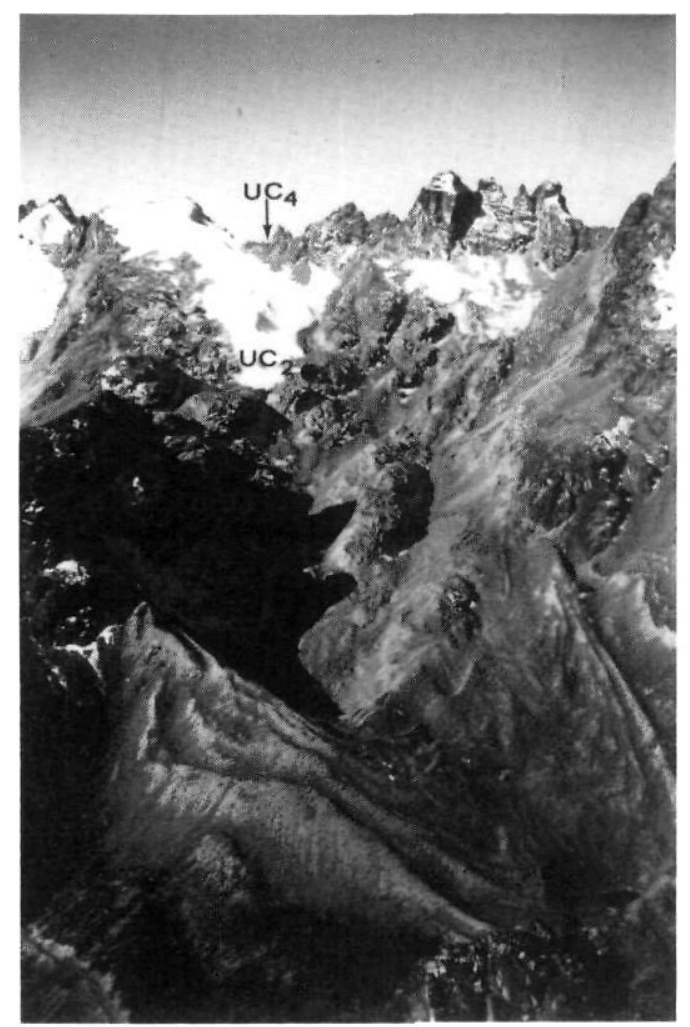

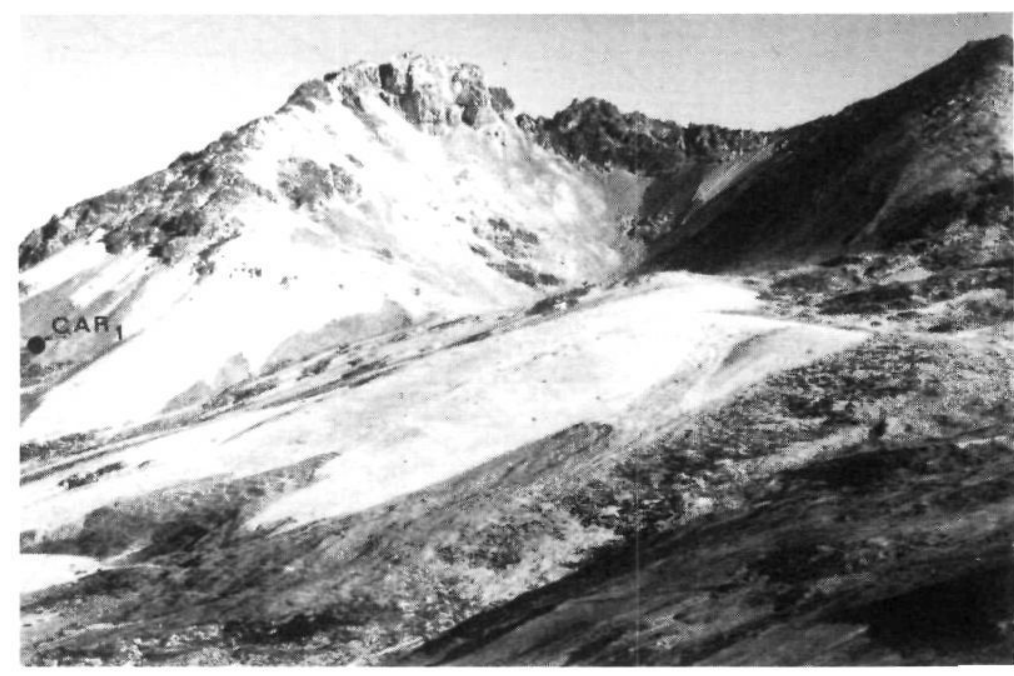

FIGURE 6. Le versant Carrizal in 1987. Point: station CAR 1.

The Carrizal slope in 1987. Black circle: CAR 1 station.

FIGURE 5. Le versant Ucrupata photographié en 1987. Point: station UC2; flèche: station UC4.

The Ucrupata slope in 1987. Black circle: UC2 station; arrow: UC4 station. 
lobes, de coulées et de sols figurés où l'on a pratiqué des mesures de déplacement; on doit ajouter que dans les Andes, sous cette latitude, les versants $\mathrm{S}$ à $\mathrm{W}$ ont un microclimat plus froid et plus humide, comme l'exprime très bien le niveau de la glaciation qui y est déprimé jusqu'à $5000 \mathrm{~m}$;

b) Le versant Carrizal (fig. 4 et 6), d'orientation NE, est dépourvu de glacier jusqu'à son sommet $(5300 \mathrm{~m})$ et porte une station (CAR 1) à $5000 \mathrm{~m}$, qui permet une comparaison avec son vis-à-vis (UC 2); comme tous les versants orientés au $\mathrm{N}$ et à l'E, il comporte des grands éboulis réglants passant à leur base à des lobes et à des coulées, le tout étant en place depuis au moins le début de l'Holocène (Francou, 1988b); on a délimité à $4950 \mathrm{~m}$ un périmètre où sont pratiquées des mesures dynamiques sur des sols microstriés et des lobes à front pierreux.

On fera surtout état ici des données recueillies aux deux stations de $5000 \mathrm{~m}$ (CAR 1 et UC 2) comportant chacune un thermographe Richard-Pékly à déroulement mensuel don- nant la mesure au demi degré près. Ces appareils ont fonctionné pendant 22 mois, mais les températures recueillies en 1983 ayant subi une dérive positive anormale du fait du développement d'un phénomène ENSO (El-Niño-Southern Oscillation) très puissant cette année-là dans le Pacifique, nous présentons ici seulement celles de 1982 qui sont représentatives d'une année plus normale.

La figure 7A donne les températures sous abri à $5000 \mathrm{~m}$ sur ces deux versants. On voit qu'à cette altitude, les températures de gel peuvent être atteintes chaque jour, quelle que soit la saison, tandis que les maximums restent positifs, surtout sur le versant NE (station CAR 1). On a montré dans une précédente étude (Francou, 1988b) que ce versant enregistrait des maximums de $1,5^{\circ}$ à $2,5^{\circ} \mathrm{C}$ plus élevés en moyenne que le versant opposé.

Un relevé de la fréquence des cycles et une classification des températures négatives ont été faits pour les quatre stations de Huampar (fig. 4) et Pachachaca. On constate (fig. 8) que
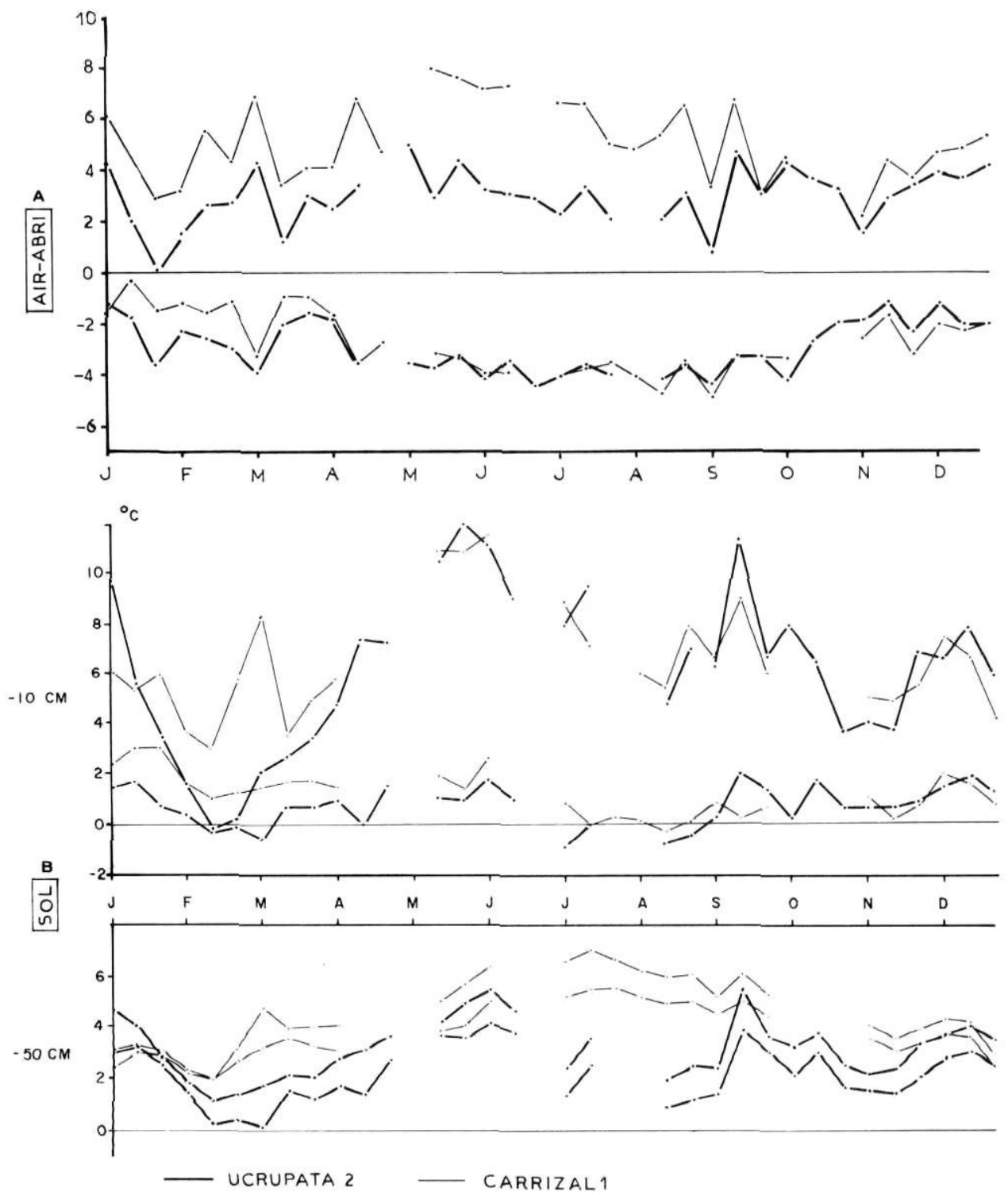

FIGURE 7. Températures maximales et minimales (en moyennes décadaires) relevées aux deux stations de $5000 \mathrm{~m}$, UC2 et CAR 1 , en 1982: A) sous abri; B) dans le sol à 10 et $50 \mathrm{~cm}$.

Maximum and minimum temperatures (10-day periods) in 1982 at the two 5,000 m stations (UC2 and CAR 1): A) in shelter; $B$ ) in the ground at 10 and $50 \mathrm{~cm}$. 


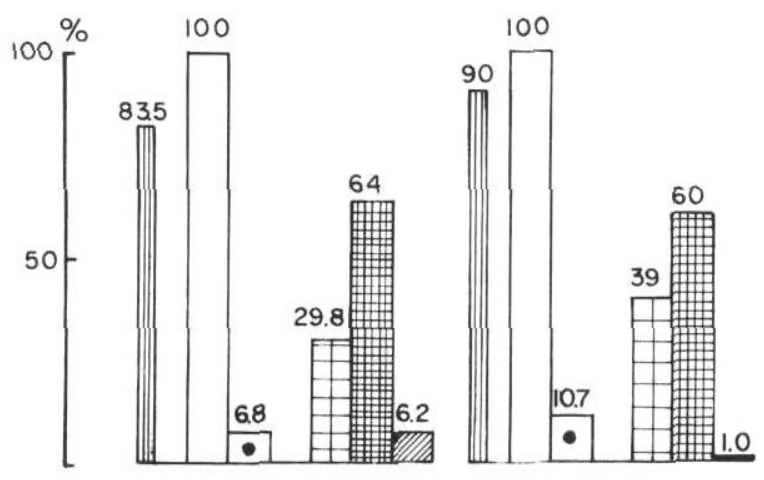

UCRUPATA 3. 5120
UCRUPATA 2. 5000

2.

3. $\bullet$

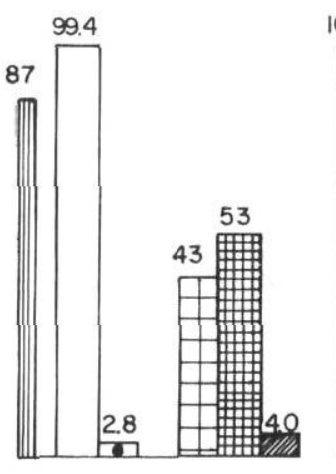

CARIZAL I. 5000

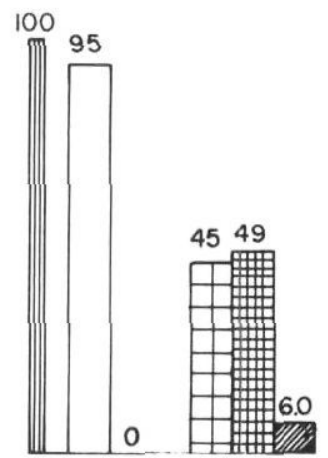

HUAMPAR 4700

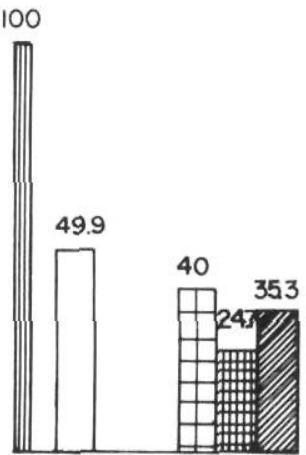

PACHACHACA 4000
१.

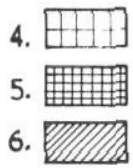

FIGURE 8. Fréquence du gel et types de gel enregistrés sous abri à Pachachaca $(4000 \mathrm{~m})$ et à Huampar aux différentes stations, en 1982: 1) nombre de jours mesurés; 2$)$ jours de gel $\left(\leq 0^{\circ} \mathrm{C}\right)$; 3 ) jours sans dégel; 4) gels compris entre $0^{\circ}$ et $2,9^{\circ} \mathrm{C} ; 4$ ) gels compris entre $-3^{\circ}$ et $-5^{\circ} \mathrm{C} ; 6$ ) gels plus bas que $-5^{\circ} \mathrm{C}$.

la probabilité du gel est maximale à partir de $4800 \mathrm{~m}$ et que ce sont les gels de faible intensité $\left(>-5^{\circ} \mathrm{C}\right)$, qui dominent largement, au moins jusqu'à $5200 \mathrm{~m}$.

\section{III-LES CARACTÉRISTIQUES DES TEMPÉRATURES NÉGATIVES RELEVÉES À HUAMPAR DANS LES PREMIERS CENTIMĖTRES DU SOL}

Deux thermosondes Richard-Pékly ont été installées dans les deux stations de $5000 \mathrm{~m}$, avec un capteur à $10 \mathrm{~cm}$ et un autre à $50 \mathrm{~cm}$ de profondeur. L'adjonction de sondes platine a permis de faire un contrôle instantané de la mesure avec un thermomètre numérique de poche et de maintenir l'erreur de la mesure proche de $\pm 0,5^{\circ} \mathrm{C}$. Les sols étudiés sont des sols d'éboulis comportant des cailloux enrobés dans une matrice sablo-silteuse et ayant tendance à s'organiser en lobes à front pierreux sur des pentes comprises entre 10 et $15^{\circ}$.

Les données sont présentées en moyennes décadaires à la figure 7B et la typologie du "gel» (on parlera ici par commodité de gel dès que la température atteint le $0^{\circ} \mathrm{C}$ ) en fonction des températures négatives atteintes à $10 \mathrm{~cm}$ de profondeur apparaît dans le tableau I. II ressort de ces données, comme cela a déjà été noté dans des milieux analogues (Schubert, 1979; Pérez, 1984), que la fréquence du gel et son intensité diminuent très vite dans les premiers centimètres du sol. Ici, sur le versant le plus froid (Ucrupata), sur 100 cycles de gel-dégel enregistrés en surface, il n'y en a qu'une quinzaine qui peuvent geler l'eau du sol à $10 \mathrm{~cm}$ de profondeur. Les cycles superficiels étant quotidiens, on peut dénombrer vers $10 \mathrm{~cm}$ de profondeur, une cinquantaine de cycles par an qui sont efficaces si la teneur en eau dans le sol est suffisante. Mais ce nombre de cycles efficaces est certainement encore moins élevé, car les températures les plus basses
Frequency of freeze-thaw cycles and type of frost from screen temperature measurements in 1982 in Huampar and Pachachaca: 1) number of measurement days; frost days ( $\leq 0^{\circ} \mathrm{C}$ ); days without thaw; 4) days with frost intensity between $0^{\circ}$ and $2.9^{\circ} \mathrm{C}$; 5) days with frost intensity between $-3^{\circ}$ and $-5^{\circ} \mathrm{C}$; 6) days with frost intensity below $5^{\circ} \mathrm{C}$.

atteintes à cette profondeur du sol le sont pendant les mois secs où la recharge en eau du sol n'est pas assurée régulièrement. En versant $\mathrm{NE}$, plus sec, le nombre de ces cycles efficaces tombe certainement en dessous de 20 à cette profondeur. Néanmoins, d'autres études ont montré que les conditions de pénétration du front de gel dans le sol ne sont pas identiques partout.

\section{IV - LE RÔLE DE LA GRANULOMÉTRIE ET DES TENEURS EN EAU DU SOL DANS LA FRÉQUENCE ET L'INTEN- SITÉ DU GEL}

a) La granulométrie

Deux thermomètres à maximum-minimum ont été introduits dans le sol à proximité des sondes: le premier à $5 \mathrm{~cm}$ de profondeur dans la bande fine (sables, silts) d'un sol strié, l'autre à la même profondeur mais dans la cloison graveleuse openwork. Le tableau II comporte aussi les données correspondantes obtenues à 10 et $50 \mathrm{~cm}$ de profondeur grâce à la thermosonde dans le sol hétérométrique.

II ressort que la pénétration du front de gel est bien meilleure avec une granulométrie fine: les températures minimales atteintes y sont de 2 à $4^{\circ} \mathrm{C}$ inférieures aux autres, sauf à une période, la plus humide, où le sol a été maintenu saturé en permanence (février-mars 1983). On comprend dans ces conditions comment peuvent se faire la croissance des glaces d'exsudation et le gonflement que l'on observe sur les bandes fines de ces sols striés ou sur les taches de terre (nubbins).

b) Les teneurs en eau

D'autre part, des mesures de températures ont été faites avec un thermographe Grant à mémoire de type Squirrel de 
TABLEAU ।

Le gel dans le sol à $10 \mathrm{~cm}$ de profondeur à $5000 \mathrm{~m}$ d'altitude aux stations UC2 et CAR1 (1982);

gel $=$ température $\leqslant 0^{\circ} \mathrm{C}$

\begin{tabular}{|c|c|c|c|c|c|c|c|}
\hline UC2 & $\begin{array}{l}\text { Nombre de } \\
\text { jours de gel }\end{array}$ & $\begin{array}{l}\text { Nombre } \\
\text { de cycles }\end{array}$ & $\begin{array}{l}\text { Gels } \\
\text { à } 0^{\circ} \mathrm{C}\end{array}$ & $\begin{array}{c}\text { Gels } \\
\text { à }-1^{\circ} \mathrm{C}\end{array}$ & $\begin{array}{c}\text { Gels } \\
\text { à }-2^{\circ} \mathrm{C}\end{array}$ & $\begin{array}{l}\text { Sans } \\
\text { dégel }\end{array}$ & $\begin{array}{l}\text { Nombre } \\
\text { de jours }\end{array}$ \\
\hline Janvier & 07 & 06 & 06 & 00 & 01 & 01 & 31 \\
\hline Février & 22 & 02 & 17 & 05 & 00 & 21 & 25 \\
\hline Mars & 19 & 08 & 12 & 07 & 00 & 08 & 31 \\
\hline Avril & 11 & 12 & 08 & 04 & 00 & 00 & 28 \\
\hline Mai & 05 & 05 & 04 & 01 & 00 & 00 & 24 \\
\hline Juin & 11 & 11 & 09 & 02 & 00 & 00 & 24 \\
\hline Juillet & 20 & 20 & 10 & 08 & 02 & 00 & 21 \\
\hline Août & 23 & 23 & 12 & 09 & 02 & 00 & 24 \\
\hline Septembre & 09 & 09 & 07 & 02 & 00 & 00 & 27 \\
\hline Octobre & 11 & 11 & 08 & 02 & 01 & 00 & 31 \\
\hline Novembre & 15 & 14 & 13 & 02 & 00 & 01 & 30 \\
\hline Décembre & 05 & 05 & 05 & 00 & 00 & 00 & 31 \\
\hline Total & 158 & 126 & 111 & 42 & 06 & 30 & 327 \\
\hline$\%$ & 48 & 38,5 & 70 & 26 & 04 & 19,6 & 90 \\
\hline \multicolumn{8}{|l|}{ CAR1 } \\
\hline Janvier & 00 & 00 & 00 & 00 & 00 & 00 & 31 \\
\hline Février & 01 & 01 & 01 & 00 & 00 & 00 & 20 \\
\hline Mars & 05 & 05 & 05 & 00 & 00 & 00 & 31 \\
\hline Avril & 00 & 00 & 00 & 00 & 00 & 00 & 14 \\
\hline Mai & 00 & 00 & 00 & 00 & 00 & 00 & 13 \\
\hline Juin & 01 & 01 & 01 & 00 & 00 & 00 & 11 \\
\hline Juillet & 20 & 20 & 17 & 03 & 00 & 00 & 31 \\
\hline Août & 20 & 20 & 12 & 08 & 00 & 00 & 29 \\
\hline Septembre & 14 & 14 & 13 & 01 & 00 & 00 & 30 \\
\hline Octobre & 02 & 02 & 01 & 01 & 00 & 00 & 05 \\
\hline Novembre & 10 & 09 & 08 & 02 & 00 & 01 & 30 \\
\hline Décembre & 06 & 06 & 05 & 01 & 00 & 00 & 31 \\
\hline Total & 79 & 78 & 63 & 16 & 00 & 01 & 276 \\
\hline$\%$ & 29 & 28 & 80 & 20 & 00 & 01 & 76 \\
\hline
\end{tabular}

TABLEAU ॥

Températures extrêmes relevées sous abri, à 5,10 et $50 \mathrm{~cm}$ de profondeur dans le sol à la station UC2 à $5000 \mathrm{~m}$ (septembre 1982 à octobre 1983)

\begin{tabular}{|c|c|c|c|c|c|c|c|c|c|c|}
\hline & \multicolumn{2}{|c|}{ Abri } & \multicolumn{2}{|c|}{$-5 \mathrm{~cm}$ fines } & \multicolumn{2}{|c|}{$-5 \mathrm{~cm}$ graviers } & \multicolumn{2}{|c|}{$-10 \mathrm{~cm}(F+G)$} & \multicolumn{2}{|c|}{$-50 \mathrm{~cm}(\mathrm{~F}+\mathrm{G})$} \\
\hline & Tx & $\mathrm{Tn}$ & Tx & Tn & $T x$ & Tn & $T x$ & $\mathrm{Tn}$ & $T x$ & $\mathrm{Tn}$ \\
\hline $27.08-29.09 .82$ & - & $-5,0$ & - & $-6,0$ & 19,0 & $-3,0$ & 14,0 & 0,0 & 7,0 & 0,0 \\
\hline $29.09-28.10 .82$ & 9,0 & $-7,0$ & 26,0 & $-5,0$ & 21,0 & $-3,0$ & 13,0 & $-2,0$ & 5,0 & 1,0 \\
\hline $28.10-26.11 .82$ & 6,0 & $-5,0$ & - & - & - & - & 8,0 & $-1,0$ & 4,0 & 0,0 \\
\hline $26.11-06.01 .83$ & 9,0 & $-4,0$ & 18,0 & $-5,0$ & 25,0 & $-1,0$ & 12,0 & $-0,0$ & 7,0 & 0,0 \\
\hline $06.01-15.02 .83$ & 10,0 & $-5,0$ & 12,0 & $-3,0$ & 28,0 & $-1,0$ & 19,0 & 1,0 & 10,0 & 3,0 \\
\hline $15.02-21.04 .83$ & 9,5 & $-3,5$ & 20,0 & 0,0 & 22,0 & $-1,0$ & 12,0 & $-1,0$ & 9,0 & 1,0 \\
\hline $21.04-08.06 .83$ & 9,0 & $-6,0$ & 22,0 & $-4,0$ & 36,0 & $-1,0$ & 12,0 & $-2,0$ & 6,0 & 2,0 \\
\hline $08.06-08.07 .83$ & 8,0 & $-6,0$ & 20,0 & $-4,0$ & 20,0 & $-2,0$ & 14,0 & $-2,0$ & 6,0 & 0,0 \\
\hline 08.07-15.08.83 & 9,0 & $-7,0$ & 27,0 & $-4,0$ & 21,0 & $-3,0$ & 14,0 & 0,0 & 7,0 & 3,0 \\
\hline $15.08-17.09 .83$ & 8,5 & $-6,0$ & 28,0 & $-3,5$ & 23,0 & $-1,0$ & 12,0 & $-1,0$ & 5,0 & 2,0 \\
\hline $17.09-05.10 .83$ & 9,0 & $-4,0$ & 22,0 & $-4,0$ & - & - & 11,0 & 0,0 & 5,0 & 3,0 \\
\hline Moyenne & 8,7 & $-5,4$ & 21,6 & $-3,8$ & 23,9 & $-1,7$ & 12,8 & $-0,7$ & 6,4 & 1,4 \\
\hline
\end{tabular}

Fines: sol sablo-silteux; Graviers: gravier à texture ouverte; $F+G$ : sol hétérométrique. 
$0,3^{\circ} \mathrm{C}$ de résolution muni de quatre thermistances pendant plusieurs jours en juillet 1985 et juillet 1987 sur le bord d'un lac à $4860 \mathrm{~m}$, en terrain morainique et avec une pente de moins de $10^{\circ}$; elles ont donné les informations suivantes (Francou, 1986; Francou et Bourgeat, 1988):

- sur les berges du lac où se forment chaque jour des pipkrakes de $5 \mathrm{~cm}$, la température minimale du sol ne dépasse pas $-4^{\circ} \mathrm{C}$ à $5 \mathrm{~cm}$ de profondeur et le front de gel n'atteint pas $10 \mathrm{~cm}$, ceci par des températures voisines de $-10^{\circ} \mathrm{C}$ à la surface du sol;

- dès que l'on s'éloigne des berges du lac, au-delà de l'auréole où la proximité de la nappe phréatique permet aux pipkrakes de se former, le front de gel peut descendre à $10 \mathrm{~cm}$ de profondeur et même un peu en dessous.

Cette observation permet donc de différencier un milieu de versant bien égoutté, où le gel peut pénétrer dans le sol sur $10-15 \mathrm{~cm}$ et les zones mal drainées où la couche affectée par le gel ne dépasse pas $5 \mathrm{~cm}$. Dans le premier peuvent se développer d'autres sortes de ségrégations de glace que celles conduisant à la formation des pipkrakes, comme nous le verrons plus loin.

\section{V - LES TEMPÉRATURES DANS LES PAROIS RO- CHEUSES EN DESSOUS DE $5200 \mathrm{M}$}

a) Les conditions de saisie des températures dans la roche

Une thermosonde multivoie de marque Grant a été installée sur une petite paroi d'andésite à $5150 \mathrm{~m}$ d'altitude (station UC4, fig. 4). Cet appareil est doté de sondes platine de 100 ohms donnant une précision théorique de $0,1^{\circ} \mathrm{C}$, mais qui n'est pas en fait meilleure que $0,5^{\circ} \mathrm{C}$ en raison du dépouillement manuel sur table à digitaliser. Les sondes ont été placées dans des trous forés à $2 \mathrm{~cm}$ sur des facettes orientées au NE et au SW, dans une fissure de $0,5 \mathrm{~cm}$ de diamètre à 2 et $25 \mathrm{~cm}$ de profondeur et à la surface même du rocher. Le contact avec le matériel rocheux est assuré par un mélange de graisse et de poudre d'aluminium. La sonde surface est protégée du rayonnement solaire direct par un mélange de résine et de roche pulvérisée. Un écran en bois abrite une sonde dite air éloignée de $20 \mathrm{~cm}$ du rocher (Francou, 1988a). Malheureusement, par suite de difficultés techniques, on n'a pu obtenir que trois séquences d'enregistrement, chacune, par chance, étant centrée sur trois types de temps contrastés et représentatifs.

\section{b) Les résultats}

Le tableau III regroupe les principales caractéristiques de l'enregistrement de juin-juillet 1983, mois qui ont été, rappelonsle, $1^{\circ} \mathrm{C}$ environ plus chauds que la normale à Pachachaca (Francou, 1988b). Les tableaux IV et $V$ font de même pour les plus courtes séquences de septembre 1983 et de novembre-décembre 1982 qui, au contraire de juin-juillet, correspondent à des épisodes de précipitations.

Malgré leur caractère partiel, ces données soulignent les faits suivants:

- le gel dans la roche, comme dans les sols, est de faible intensité et de courte durée jusqu'à 5200 m; la température reste toujours supérieure à $-5^{\circ} \mathrm{C}$ dans les premiers centimètres et l'échauffement systématique de la journée exclut la persistance de températures négatives pendant plusieurs jours;

- la durée et l'intensité du gel augmentent nettement dans la roche lors des épisodes de temps perturbé, comme le montrent les données obtenues en septembre et surtout en décembre; dans ce contexte humide marqué par des chutes de neige fréquentes, le refroidissement du massif rocheux et l'imbibition du milieu poreux par les eaux de fonte de la neige permettent des minimums plus bas, alors que dans le même temps, les minimums thermiques relevés sous abri sont plus doux que par temps $\sec \left(-2 /-3^{\circ} \mathrm{C}\right.$ contre $\left.-5 /-6^{\circ} \mathrm{C}\right)$. On s'est basé sur ces enregistrements pour déterminer les paramètres durée-intensité de gel d'un protocole de gélifraction expérimentale auquel on a soumis des échantillons provenant de ces parois rocheuses (voir infra). Pour être complet, signalons enfin que des mesures similaires ont été effectuées à $5500 \mathrm{~m}$ en Cordillère blanche sur une granodiorite, au Nevado Vallunaraju (Francou, 1988a): elles ont démontré l'existence du pergélisol dans

\section{TABLEAU III}

Températures enregistrées à la station UC4 à $5150 \mathrm{~m}$ en paroi sur six sondes en juin-juillet 1983

Air Surface $-2 \mathrm{~cm}-2 \mathrm{~cm}$ Fissure Fissure NE SW $-2 \mathrm{~cm} \mathrm{NE}-25 \mathrm{~cm}$

\begin{tabular}{|c|c|c|c|c|c|c|}
\hline $\begin{array}{l}\text { Cycles de gel- } \\
\text { dégel }\end{array}$ & 36 & 14 & 03 & 17 & 04 & 0 \\
\hline Minimum absolu & $-3,8$ & $-1,8$ & $-2,5$ & $-1,1$ & $-2,6$ & 5,0 \\
\hline Moyenne des & $-1,6$ & 0,4 & 1,0 & $-0,1$ & 1,0 & 7,1 \\
\hline $\begin{array}{l}\text { minimums et } \\
\text { écarts types }\end{array}$ & 0,7 & 1,0 & 1,0 & 0,7 & 1,0 & 0,8 \\
\hline $\begin{array}{l}\text { Heures de gel } \\
\text { (interpolation) }^{1}\end{array}$ & 294 & 51 & 10 & 98 & 11 & 0 \\
\hline $\begin{array}{l}\text { Heures de gel } \\
\text { par jour }\end{array}$ & 9,8 & 1,7 & 0,3 & 3,3 & 0,3 & 0 \\
\hline Durée maximale & 20 & 12 & 08 & 12 & 08 & 0 \\
\hline
\end{tabular}
de gel continu (heures)

\begin{tabular}{lrrrrrr} 
Maximum absolu & 10,6 & 15,1 & 23,8 & 20,5 & 23,9 & 9,9 \\
$\begin{array}{l}\text { Moyenne des } \\
\text { maximums et }\end{array}$ & 7,0 & 10,6 & 18,0 & 13,1 & 18,0 & 8,4 \\
écarts types & 2,5 & 3,7 & 4,5 & 3,8 & 0,9 \\
$\begin{array}{l}\text { Amplitude } \\
\text { moyenne }\end{array}$ & 8,6 & 10,2 & 17,0 & 13,2 & 17,0 & 1,3 \\
$\begin{array}{l}\text { Minimum absolu } \\
\text { des }\end{array}$ & $-4,2$ & $-2,5$ & $-2,5$ & $-2,1$ & $-2,6$ & 2,8 \\
$\begin{array}{l}\text { enregistrements } \\
\text { (5 mois) }\end{array}$ & & & & & & \\
\hline
\end{tabular}

1. Calculées par interpolation : le gel est supposé avoir commencé une heure avant son enregistrement et s'être terminé une heure après son dernier enregistrement. Les gels isolés "valent" une heure. 
TABLEAU IV

Températures enregistrées à la station UC4 à $5150 \mathrm{~m}$ en paroi lors de la troisième décade de septembre 1983

\begin{tabular}{|c|c|c|c|c|c|c|}
\hline & Air & Surface & $\begin{array}{l}-2 \mathrm{~cm} \\
\mathrm{NE}\end{array}$ & $\begin{array}{c}-2 \mathrm{~cm} \\
\mathrm{SW}\end{array}$ & $\begin{array}{l}\text { Fissure } \\
-2 \mathrm{~cm}\end{array}$ & $\begin{array}{l}\text { Fissure } \\
-25 \mathrm{~cm}\end{array}$ \\
\hline $\begin{array}{l}\text { Cycles de gel- } \\
\text { dégel }\end{array}$ & 11 & 08 & 08 & 06 & 07 & 0 \\
\hline Minimum absolu & $-2,8$ & $-1,3$ & $-1,4$ & $-0,8$ & $-1,4$ & 3,7 \\
\hline $\begin{array}{l}\text { Moyenne des } \\
\text { minimums et } \\
\text { écarts types }\end{array}$ & $\begin{array}{r}-2,0 \\
0,4\end{array}$ & $\begin{array}{r}-0,5 \\
0,5\end{array}$ & $\begin{array}{r}-0,5 \\
0,6\end{array}$ & $\begin{array}{r}-0,1 \\
0,4\end{array}$ & $\begin{array}{r}-0,5 \\
0,6\end{array}$ & $\begin{array}{l}4,3 \\
0,5\end{array}$ \\
\hline $\begin{array}{l}\text { Heures de gel } \\
\text { (interpolation) }\end{array}$ & 103 & 27 & 25 & 25 & 25 & 0 \\
\hline $\begin{array}{l}\text { Heures de gel } \\
\text { par jour }\end{array}$ & 10,3 & 2,7 & 2,5 & 2,5 & 2,5 & 0 \\
\hline $\begin{array}{l}\text { Durée maximale } \\
\text { de gel continu } \\
\text { (heures) }\end{array}$ & 18 & 06 & 06 & 08 & 06 & 0 \\
\hline Maximum absolu & 7,6 & 9,1 & 16,8 & 12,0 & 16,8 & 6,3 \\
\hline $\begin{array}{l}\text { Moyenne des } \\
\text { maximums et } \\
\text { écarts types }\end{array}$ & $\begin{array}{l}5,1 \\
1,5\end{array}$ & $\begin{array}{l}6,9 \\
1,1\end{array}$ & $\begin{array}{r}13,0 \\
3,1\end{array}$ & $\begin{array}{l}9,3 \\
1,4\end{array}$ & $\begin{array}{r}12,3 \\
3,5\end{array}$ & $\begin{array}{l}5,5 \\
0,4\end{array}$ \\
\hline $\begin{array}{l}\text { Amplitude } \\
\text { moyenne }\end{array}$ & 7,1 & 7,3 & 13,5 & 9,4 & 12,8 & 1,2 \\
\hline
\end{tabular}

la roche à cette altitude, ce qui n'est pas le cas à Huampar jusqu'à $5200 \mathrm{~m}$.

Sur la base de ces données provenant de différents sites, on peut présenter une image globale du régime thermique de cette partie des Andes en soulignant les acquis suivants:

1. La fréquence des cycles de gel-dégel devient maximale à partir de $4800 \mathrm{~m}$.

2. Dans cette zone assez humide qui reçoit $800 \mathrm{~mm}$ d'eau répartis sur 170 jours en moyenne, le gel de l'eau dans les premiers centimètres du sol peut intervenir à raison d'un jour sur deux environ.

3. La tranche de sol parcourue régulièrement par le front de gel est comprise entre 5 et $10 \mathrm{~cm}$, suivant les conditions de drainage, de granulométrie et d'exposition.

4. En raison de la forte insolation de la mi-journée, le régime du gel reste rythmé par le cycle journalier; cependant, le maintien du sol à une température légèrement négative pendant plus d'une journée en exposition SW à la suite de la persistance de la neige (cas de la deuxième décade de février en 1982, cf. la fig. 7 B), laisse supposer qu'une péjoration des conditions cryo-nivales actuelles suffirait à développer des gélisols sur une, voire plusieurs décades consécutives. On sait qu'avec le cycle journalier, cela a été montré par les mesures faites par Fahey (1972) dans les Rocheuses, le front de gel ne peut dépasser 10-15 cm de profondeur; a contrario, la formation de la glace de ségrégation dans le sol en dessous de $20 \mathrm{~cm}$ ne peut se faire qu'avec des gels établis sur plusieurs
TABLEAU $V$

Températures enregistrées à la station UC4 à $5150 \mathrm{~m}$ en paroi entre le 27 novembre et le 3 décembre 1982

\begin{tabular}{|c|c|c|c|c|c|}
\hline & Air & Surface & $\begin{array}{l}-2 \mathrm{~cm} \\
\mathrm{NE}\end{array}$ & $\begin{array}{c}-2 \mathrm{~cm} \\
\mathrm{SW}\end{array}$ & $\begin{array}{l}\text { Fissure } \\
-25 \mathrm{~cm}\end{array}$ \\
\hline Moyenne de gel-dégel & 07 & 07 & 07 & 07 & 05 \\
\hline Minimum absolu & $-5,2$ & $-3,3$ & $-3,3$ & $-3,7$ & $-1,5$ \\
\hline $\begin{array}{l}\text { Moyenne des minimums } \\
\text { et écarts types }\end{array}$ & $\begin{array}{r}-3,5 \\
0,9\end{array}$ & $\begin{array}{r}-2,3 \\
0,7\end{array}$ & $\begin{array}{r}-1,4 \\
1,0\end{array}$ & $\begin{array}{r}-2,0 \\
1,2\end{array}$ & $\begin{array}{r}-0,2 \\
0,9\end{array}$ \\
\hline $\begin{array}{l}\text { Heures de gel } \\
\text { (interpolation) }\end{array}$ & 114 & 67 & 46 & 70 & 31 \\
\hline Heures de gel par jour & 16,3 & 9,6 & 6,6 & 10 & 4,4 \\
\hline $\begin{array}{l}\text { Durée maximale de gel } \\
\text { continu (heures) }\end{array}$ & 22 & 14 & 12 & 14 & 10 \\
\hline Maximum absolu & 6,6 & 8,2 & 20,4 & 6,8 & 5,7 \\
\hline Moyenne des & 3,6 & 5,5 & 10,2 & 4,8 & 3,5 \\
\hline $\begin{array}{l}\text { maximums et écarts } \\
\text { types }\end{array}$ & 2,4 & 2,0 & 6,3 & 1,0 & 1,5 \\
\hline Amplitude moyenne & 7,1 & 7,8 & 11,6 & 8,8 & 3,7 \\
\hline
\end{tabular}

jours, condition qui paraît exceptionnelle, sinon impossible, dans le contexte actuel sur cette partie des Andes. Les expériences de gel faites au Centre de géomorphologie à partir de sols sablo-silteux ayant une teneur en eau moyenne, montrent qu'il faut un gel continu sur une semaine à $-10^{\circ} \mathrm{C}$ pour que le front de gel atteigne $30 \mathrm{~cm}$ de profondeur.

\section{LE RÔLE DU GEL DANS LES DYNAMIQUES DE VERSANT}

\section{I - LE GEL ET LA FRAGMENTATION DE LA ROCHE}

La zone de Huampar appartient géologiquement au volcanisme Pacococha constitué pour l'essentiel d'andésites, de dacites et de rhyolites recoupées par des intrusifs, le tout d'âge néogène (Mégard, 1973). L'intensité de la fracturation et l'importance des phénomènes d'altération confèrent à cet ensemble une forte sensibilité vis-à-vis des agents de fragmentation, comme en témoigne la tendance qu'ont de nombreuses corniches à se faire régulariser (Dollfus, 1965; Francou, 1988b).

On a pu cerner l'efficacité actuelle de la gélifraction de trois façons:

a) Des blocs déposés par les glaciers en recul ont complètement éclaté sur place; à titre d'exemple, on peut citer le cas d'un bloc de $0,3 \mathrm{~m}^{3}$ abandonné par le glacier Ucrupata il y a trente ans d'après la couverture photographique aérienne de 1951 et qui a été débité en éléments de 0,1 m de longueur au maximum; en supposant que le débitage ait commencé lors du dépôt par le glacier, on peut évaluer à 10000 le nombre de cycles reçus, chiffre qui correspond, au maximum, à 4 000-5 000 cycles ayant pu entraîner la formation de la glace dans le bloc; ces blocs que l'on trouve éclatés portent tous la marque d'une forte altération, la plupart du 
temps par épidotisation, et se classent parmi les échantillons comportant une forte porosité que l'on situe entre 10 et $20 \%$ (Francou, 1988b).

b) Par contre, la grande majorité des blocs moins altérés qui constituent les éboulis mis en place depuis le Tardiglaciaire sur les versants $\mathrm{N}$ à $\mathrm{E}$ sont recouverts d'une patine d'oxydation ferrugineuse; on ignore la rapidité de mise en place de cette patine, mais le fait que les tranchées des routes ouvertes il y a plus de cinquante ans n'en soient pas recouvertes prouve qu'il s'agit d'un phénomène lent, d'ordre séculaire ou peutêtre millénaire; la rareté des chutes de pierres observées sur ce même type de versant (Francou, 1988c) va dans le même sens et témoigne de la faible activité de ces parois actuellement.

c) II en va tout autrement des corniches $\mathrm{S}$ à $\mathrm{W}$ qui ont connu depuis 50 ans un rétrécissement rapide de leurs surfaces englacées; ainsi pour la paroi rocheuse dominant le glacier Ucrupata et portant la station UC4 (Fig. 4 et 9), l'estimation du volume des débris tombés sur la glace durant l'année 1982 nous a permis de fixer l'ordre de grandeur du recul de cette paroi très active: il est de l'ordre de $2-3$ mm.an $^{-1}$; le débitage accéléré des corniches rocheuses en voie de déglacement est un fait reconnu et souvent souligné (Church et Ryder, 1972; Whalley, 1974; Tricart et Cailleux, 1965; Hétu, 1986); même si les mécanismes en présence ne sont pas parfaitement expliqués, on sait que ces derniers relèvent davantage de facteurs endogènes (relâchement des contraintes, modification des efforts exercés sur le réseau fissural existant) que des processus de météorisation.

La conclusion que l'on peut tirer de ces observations est que, mis à part les faciès les plus gélifs, la gélifraction est aujourd'hui peu active en dessous de $5200 \mathrm{~m}$; les parois les plus actives sont celles qui sont en phase de réadaptation morphoclimatique par suite de la disparition rapide des zones englacées. Une vérification expérimentale est en cours au Centre de géomorphologie avec un protocole de "gel doux" $\left(-3,5 /+11^{\circ} \mathrm{C}\right)$ et rapide. Les premiers résultats (non publiés) montrent que seuls les faciès très poreux se fragmentent, ce qui tend à accréditer l'hypothèse selon laquelle la fourniture des éléments très grossiers des éboulis n'a pu intervenir qu'avec un contexte climatique plus rigoureux que l'actuel, dans lequel les gels étaient plus efficaces.

\section{I - LA GLACE DE SÉGRÉGATION DANS LES SOLS ET LE RUISSELLEMENT DIFFUS}

À partir des observations directes et de l'analyse micromorphologique, trois types de glaces de ségrégation ont pu être distingués dans ce milieu:

a) Les pipkrakes: ils rencontrent ici des conditions de développement optimales: baisse rapide des températures en fin de journée, températures atteignant vite au sol le niveau des $-8 /-10^{\circ} \mathrm{C}$, bonne alimentation en eau dans les zones déprimées et en aval des taches de neige fondant lentement en saison sèche, porosité optimale des sols, comprise entre 50 et $60 \%$ (Outcalt, 1971; Meetmayer et Zippin, 1981); comme on l'a vu, là où ils se forment, la pénétration du front de gel ne dépasse pas $5 \mathrm{~cm}$ de profondeur; à Huampar (voir infra),

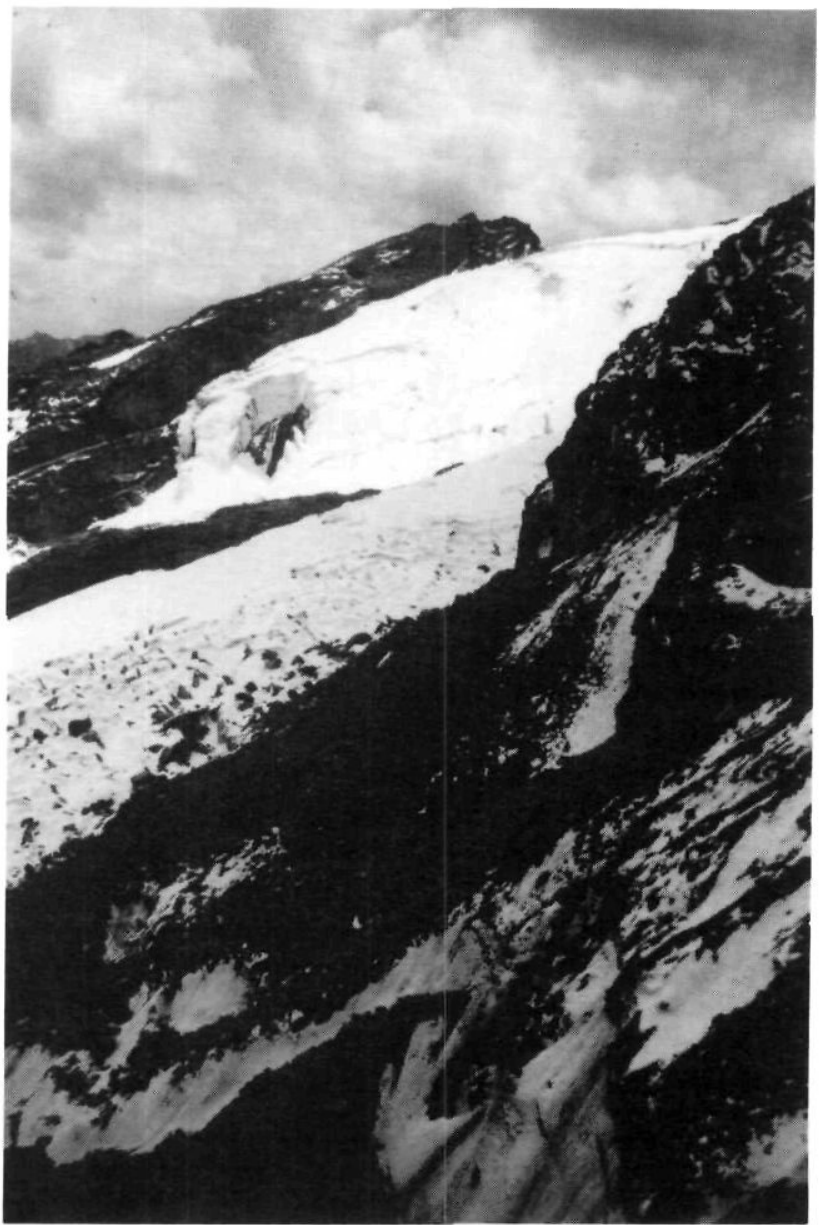

FIGURE 9. Chutes de pierres très fréquentes sur le glacier Ucrupata témoignant d'un recul rapide de la paroi (état en octobre 1983).

Frequent rockfalls on the glacier Ucrupata which attests the high retreat of the cliff (1983).

la contribution des pipkrakes au déplacement des éléments se marque par la dispersion des valeurs obtenues sur chaque alignement et par le fait que les petits éléments, ceux dont le grand axe ne dépasse pas $10 \mathrm{~cm}$, se déplacent nettement plus vite que les plus gros (Pérez, 1987); comme cela a été souligné à maintes reprises (Pissart, 1973, notamment), le rôle des pipkrakes dans la mise en place des figures de tri est évident.

b) La cryoreptation (frost-creep): les ségrégations de forme lenticulaire ou lamellaire peuvent être observées très couramment dans le sol à partir de $5 \mathrm{~cm}$ environ de la surface, particulièrement sur les pentes bien drainées où la formation des pipkrakes n'est pas exclusive; à l'analyse micromorphologique apparaissent les plans de stratification avec cisaillements qui sont à l'origine de la cryoreptation (Van VlietLanoë et Francou, 1988); dans l'étude des déplacements, nous avons attribué le mouvement lent (moins de $5 \mathrm{~cm}$.an ${ }^{-1}$ ) et généralisé des particules à la cryoreptation; l'expulsion progressive des éléments grossiers des nappes-coulées qui conduit au litage des dépôts (voir infra) est liée aussi à ce type de ségrégation, 
c) La gélifluxion et les coulées de boue: ces déplacements rapides de type plastique sont observés en période de surcharge hydrique du sol sur les formations morainiques où la fraction fine est plus abondante; ce qui prouve que le schéma montrant le passage, avec des teneurs en eau croissantes, de la cryoreptation à la gélifluxion, puis à des écoulements boueux qui ont été décrits dans les milieux arctiques et alpins (Van Vliet-Lanoë, 1988) est valable aussi dans la haute montagne des basses latitudes.

La fusion rapide et répétée de la couche de neige et de la glace du sol provoque un ruissellement diffus qui joue un grand rôle dans le transport des sables et de la fraction fine. On ne dispose pas de mesures sur ce processus, mais une estimation faite à partir d'une couche de neige et de grésil de $15 \mathrm{~cm}$ surmontant un niveau de $5 \mathrm{~cm}$ de pipkrakes qui fondent en 3 heures sur un éboulis fin, situation optimale, donne une décharge hydrique proche de $5 \mathrm{~mm}$, avec un débit horaire maximal qui pourrait atteindre $2 \mathrm{~mm}$. II s'agit là d'une quantité d'eau inférieure à celle qui est nécessaire pour provoquer un ruissellement de dissection sur ce type de substrat (Yaïr et Lavee, 1974; Caine, 1980), mais elle est toutefois suffisante pour développer un efficace lavage des petites particules. Des figures striées superficielles de toutes tailles sont attribuées à ce processus de lavage, tout comme l'éluviation des produits matriciels qui intervient au niveau des fronts des nappes-coulées (voir infra).

\section{III - LA VITESSE DE DÉPLACEMENT DES FRAGMENTS SUR LES PENTES}

Un système de balisage a été mis en place en 1982, sur deux parcelles pour étudier le déplacement des particules. La méthode utilisée est celle des alignements de balises peintes tracés au cordeau entre deux bases fixes perpendiculairement à l'axe de la pente. Avec cette méthode, l'erreur de la mesure est au maximum de $0,5 \mathrm{~cm}$. On relève en plus la longueur de chaque fragment balisé ainsi que la nature de son substrat. Trois lignes ont été tracées sur le versant Carrizal à $4950 \mathrm{~m}$ sur une pente de $5-10^{\circ}$ comportant des figures striées et cinq lignes sur le versant Ucrupata dans un petit vallon à profil longitudinal convexe situé à $4900 \mathrm{~m}$ où la pente varie de $11^{\circ}$ en haut à $28^{\circ}$ en bas. Ce second site présente du matériel plus grossier que le premier, avec des blocs de 10 à $40 \mathrm{~cm}$ d'axe $a$.

Parmi les résultats déjà publiés (Francou, 1988b), les enseignements les plus intéressants à tirer sont les suivants:

a) sur Carrizal, la distribution des déplacements annuels est de type log-normale, ce qui est classique dans ce genre de mesures (Caine, 1963; Pérez, 1985); la médiane est de $6 \mathrm{~cm} \cdot \mathrm{an}^{-1}$ (fig. 10);

b) sur Ucrupata, la configuration du terrain permet de trouver une fonction liant le déplacement annuel et la pente (fig. 11): celle-ci est nettement de type semi-logarithmique entre le sinus de la pente et la moyenne des déplacements sur chaque ligne; la dispersion, donnée par le paramètre $\sigma$, croît également géométriquement;

c) la relation entre la taille des fragments et le déplacement parcouru à Carrizal montre clairement qu'en dessus du seuil des $10 \mathrm{~cm}$ (axe a), la valeur des déplacements ne dépasse pas $10 \mathrm{~cm} \cdot$ an $^{-1}$;

d) un mouvement lent, de l'ordre de $3-5 \mathrm{~cm} . \mathrm{an}^{-1}$ est mesuré dans les plages openwork superficielles. Ce mouvement est attribué à la glace de ségrégation qui se forme un peu plus en profondeur vers $5-10 \mathrm{~cm}$ dans les couches plus riches en matrice. On ne dispose pas pour le moment de vitesses en profondeur; toutefois on peut penser que le déplacement reste très superficiel, limité aux 5-10 premiers centimètres du sol, comme l'a montré Pérez (1985) au Venezuela.

Au total, la dispersion des valeurs obtenues au sein des alignements, la croissance géométrique de la vitesse avec la pente et l'importance que prend le facteur-taille sont caractéristiques de déplacements où les pipkrakes jouent un rôle

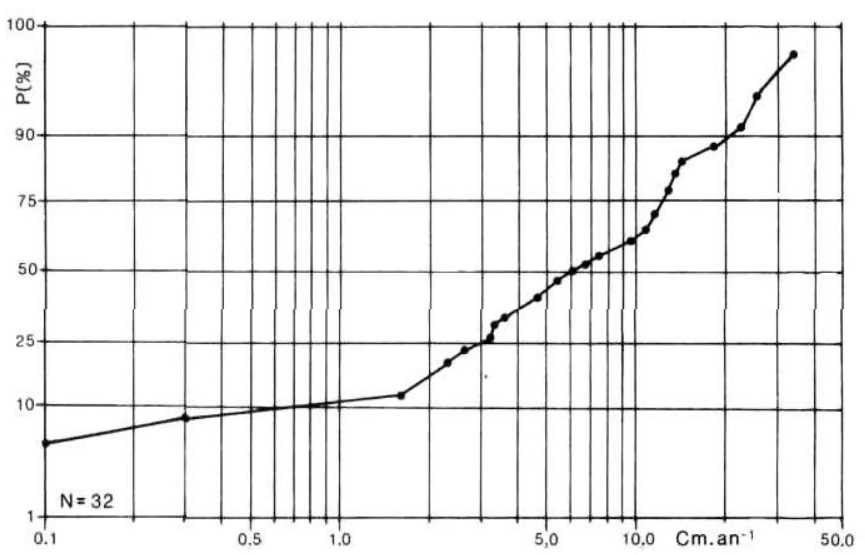

FIGURE 10. Distribution des déplacements annuels mesurés sur le périmètre de Carrizal $(4950 \mathrm{~m})$. Echelle gausso-logarithmique. Mesures de 1982 à 1985 .

Distribution of the yearly displacements measured at the Carrizal site (4950 m). Gausso-logarithmic scale. Data from 1982-1985.

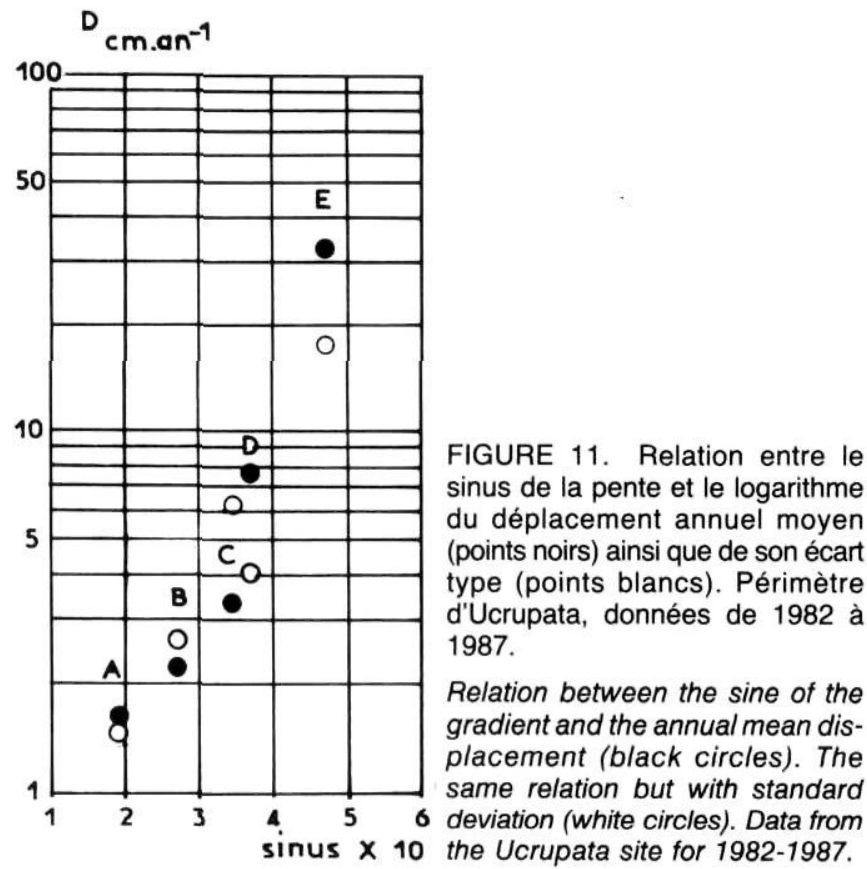


prépondérant. En cela, nos conclusions rejoignent celles de Pérez (1985 et 1987) obtenues à partir d'un milieu situé près du $10^{\circ} \mathrm{N}$.

Cependant, nous avons également la preuve ici que la cryoreptation joue aussi un rôle notable dans les dynamiques attribuées au gel, en particulier dans des sites et sédiments peu favorables à la formation des pipkrakes où un déplacement existe néanmoins.

\section{IV - LES DÉPÔTS DE PENTE STRATIFIÉS}

\section{a) Les éboulis}

Peu d'études ont été consacrées aux éboulis dans les montagnes des basses latitudes, hormis les travaux déjà cités de Pérez (1985 et 1986) au Vénézuela. En dehors des talus dont la formation actuelle, très rapide, accompagne la disparition complète des glaciers sous les corniches rocheuses et qui ont une dynamique de type gravitaire (Francou, 1988b), ces dépôts ont, dans la zone étudiée, plusieurs traits originaux par rapport à leurs équivalents alpins:

- leur profil est très tendu, avec deux valeurs modales d'inclinaison selon le type de matériel, l'une à $34-35^{\circ}$ (fig. 12), l'autre à $27-28^{\circ}$; font exception les talus à large concavité distale où fonctionnent des laves de ruissellement (debris flows);

- le classement en surface des éléments a tendance à se faire par plages isogranulométriques juxtaposées davantage que sur le mode classique de la granodécroissance longitudinale (fall sorting) rapportée par les auteurs pour les éboulis de gravité (Statham, 1976); ce trait est d'autant plus marqué que la corniche est surbaissée et que le versant tend vers le réglage;

- surtout, les talus sont très généralement stratifiés et montrent, en coupe, une alternance de lits où la matrice est dominante (matrix-supported texture) et de lits "ouverts" (openwork texture).

En utilisant les données climatiques du sol, l'étude du déplacement de marqueurs et l'analyse des coupes, on a pu aboutir à un modèle satisfaisant de la mise en place des lits (Francou, 1988c). Celle-ci est attribuée à la descente de nappes-coulées depuis le haut le long de la pente du talus, comme le montre la figure 12. La nappe comprend une importante quantité de matériel matriciel (sables, silts) et avance par cryoreptation et grâce aux pipkrakes à une vitesse annuelle évaluée à $5-10 \mathrm{~cm}$. La nappe fine est peu épaisse $(10-20 \mathrm{~cm})$ et par cryoexpulsion évacue peu à peu les cailloux et les blocs, lesquels se retrouvent en surface et finissent par alimenter le front pierreux; ils vont être ainsi incorporés à ce qui va devenir, avec l'avancée du système, le lit openwork inférieur. On voit donc dans ce modèle que le temps de mise en place complète d'un lit est long, d'ordre séculaire à millénaire et que l'installation des lits "gras" et des lits "maigres" se fait conjointement à partir de la nappe-coulée, et non par une succession de processus différents et plus ou moins cycliques comme dans les schémas classiques développés dans la littérature depuis 30 ans (Dewolf, 1988).

\section{b) Les coulées à front pierreux}

On trouve enfin sur les versants de très nombreux lobes ou coulées attribuables à la solifluxion lato sensu. Décrits par Graf (1971) sous le terme de steinzungen, ils sont d'après cet auteur les formes les plus répandues après les sols striés dans les hautes Andes péruviennes. Ils appartiennent au genre des coulées ou lobes à front pierreux (stone-banked lobes, Washburn, 1979). Contrairement aux formes décrites dans l'Arctique (Washburn, 1979) ou dans les montagnes des latitudes moyennes (Benedict, 1970), ces formes mettent en place un dépôt bien lité. Le lit fin est d'épaisseur très constante $(5-15 \mathrm{~cm}$, comme la tranche de sol parcourue par

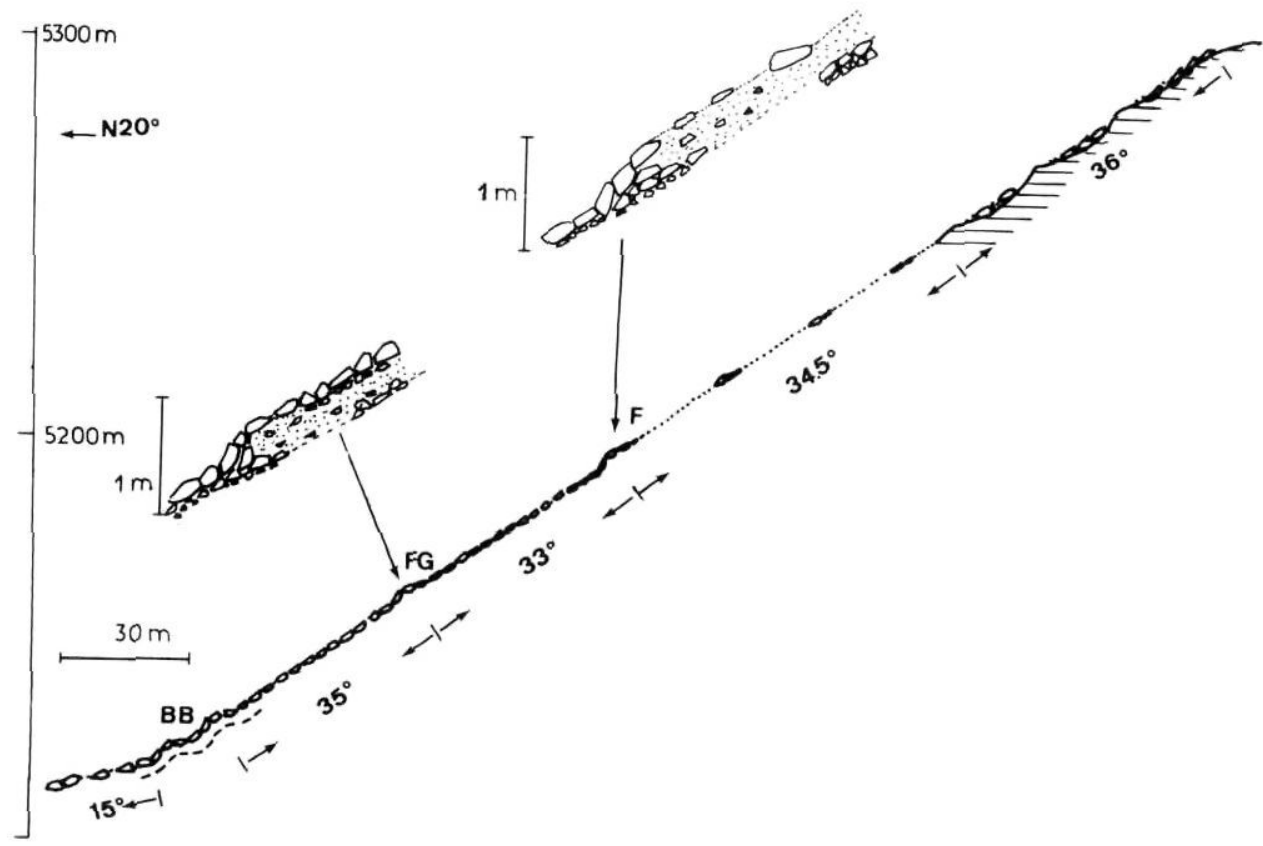

FIGURE 12. Profil de l'éboulis nord du Cerro Carrizal W et détail du front de ses nappes-coulées $(F$ et $F G$ ). Noter les bourrelets au pied.

Transect of the northward talus slope of the Cerro Carrizal W, with details of the front of two nappescoulées ( $F$ and $F G$ ). Note the basal ridges. 
le front de gel) et montre toutes les structures caractéristiques de la cryoreptation. L'origine des lits fait appel au même mécanisme décrit sur le front des nappes-coulées d'éboulis (fig. 13): expulsion des particules grossières en surface, concentration avec triage sur le front pierreux où le lavage est intense, puis basculement et ensevelissement sous l'avancée du lit fin. De tels lobes ont été traversés par des alignements de fragments peints à partir de bases fixes; on a pu y extraire les éléments suivants:

- sur le front d'un lobe situé à $4860 \mathrm{~m}$ et incliné à $6^{\circ}$, la vitesse mesurée sur deux alignements $\left(C-C^{\prime}\right.$ et $\left.B-B^{\prime}\right)$ a été entre 1985 et 1987 (Francou et Bourgeat, 1988):

C-C $: 2,8 \mathrm{~cm} \cdot \operatorname{an}^{-1}($ avec $\sigma=0,75$ pour $\mathrm{n}=10)$
B-B $^{\prime}: 1,9 \mathrm{~cm} \cdot$ an $^{-1}($ avec $\sigma=1,6$ pour $\mathrm{n}=18)$

sur ces deux lignes, des blocs de $30-40 \mathrm{~cm}$ (axe a) reposant sur le fin, ont avancé de 1 à $2 \mathrm{~cm}$.an ${ }^{-1}$;

- sur le front des coulées plus actives du versant SW à $4900 \mathrm{~m}$, les déplacements annuels ont été plus rapides: $10 \mathrm{~cm}$.an ${ }^{-1}$, pour une pente de $20^{\circ}$ (moyennes 1982-1987). Dans le cas de ces coulées plus actives, le texture du front n'est pas ouverte, mais comprend une grande quantité de produits matriciels.

\section{CONCLUSION ET DISCUSSION}

Ces différents travaux effectués sur le gel et les dynamiques de versant qui lui sont liées dans les Andes centrales, dont on n'a présenté ici qu'une synthèse rapide, permettent de déboucher sur les conclusions suivantes:

1. La fréquence du gel est maximale dès $4800 \mathrm{~m}$ et du fait du dégel qui intervient dans la matinée, le rythme du cycle de gel-dégel est journalier.

2. L'épaisseur de sol ou de roche traversée par le front de gel est faible; cette épaisseur est constante, comprise entre $5-15 \mathrm{~cm}$, mais déjà vers $10 \mathrm{~cm}$ de profondeur, le nombre des cycles décroît rapidement, ce dernier caractère est lié au rythme journalier du gel; la pénétration du gel au-delà de $15-20 \mathrm{~cm}$ ne pourrait être obtenue qu'à partir d'une diminution sensible de l'échauffement diurne.

3. Dans de telles conditions, c'est la glace d'exsudation qui se forme le plus régulièrement et l'on retrouve ses marqueurs morphodynamiques connus: déplacements à caractère individuel et très inégal des fragments sur le sol, microformes

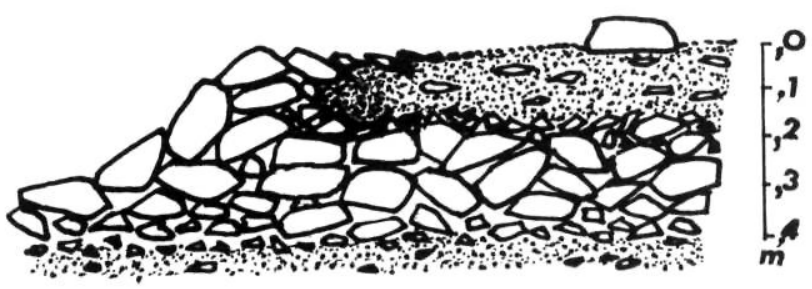

FIGURE 13. Coupe dans le front d'une coulée à front pierreux active. A noter le litage et le classement du matériel.

Active stone-banked lobe: section of the front part. Note the stratification and the sorting of particles. de tri telles que sols striés, nubbins, polygones, à l'élaboration desquelles participe conjointement le ruissellement diffus.

4. Mais la cryoreptation intervient également, ce que la littérature n'avait pas mentionné jusqu'à présent dans ce type de milieu: elle doit être prépondérante dans les sites et en des situations climatiques peu favorables à la formation des pipkrakes; on lui attribue les déplacements lents et réguliers que l'on a mesurés, la mobilité de plages grossières où la matrice éluviée est à plus de $5 \mathrm{~cm}$ de la surface, ainsi que le déplacement des nappes-coulées et des lobes dont le lit fin possède une structuration de type lamellaire.

5. Enfin, dans les conditions actuelles, le gel intervient sur les parois rocheuses sous une forme "pelliculaire"; seuls, les secteurs très altérés et microfissurés fournissent des éléments qui sont pour la plupart de la taille des sables et des graviers et viennent alimenter des nappes-coulées à granulométrie fine qui fonctionnent actuellement sur les talus.

$\mathrm{Au}$ total, il y a une bonne adéquation entre, d'une part, les caractéristiques du gel actuel que l'étude des températures nous a permis de préciser, et d'autre part, les processus que l'on a pu appréhender à partir de l'analyse des formes. Cependant, il existe plusieurs indices tendant à prouver que le cycle de gel a eu dans le passé des caractéristiques différentes:

1. Les plages à blocs patinés qui couvrent de nombreux éboulis et qui se font recouvrir actuellement par les nappescoulées à granulométrie plus fine (fig. 12 et 14) sont à mettre au compte d'une gélifraction plus intense que l'actuelle, avec des températures négatives atteintes dans la roche inférieures à $-5^{\circ}$, comme c'est le cas, à présent, au sommet du Vallunaraju à $5500 \mathrm{~m}$ en Cordillère blanche (Francou, 1988a); par voie expérimentale, on devrait prochainement fournir plus de précisions sur le seuil thermique permettant d'intensifier la gélifraction.

2. Le fonctionnement même de ces nappes-coulées grossières par cryoreptation suppose une pénétration d'un front de gel sur plus de $15-20 \mathrm{~cm}$ afin que la glace de ségrégation puisse se former dans le niveau riche en matrice; il en est de même pour les "éboulis ondulés" (Francou, 1988b) que l'on a décrits comme des talus à blocs dont le profil est accidenté de bourrelets métriques aujourd'hui inertes (fig. 15).

Que ce soit pour la gélifraction ou pour le fonctionnement cryo-induit de ces nappes-coulées et bourrelets, il a fallu un régime de gel différent, avec des températures minimales de quelques degrés plus froides et un front de gel qui puisse s'installer dans le sol sur plusieurs jours, voire plusieurs semaines. Pour réunir ces conditions, il a fallu le maintien d'une forte nébulosité dans la journée et l'existence fréquente d'un manteau neigeux peu épais mais durable.

Dans les études consacrées aux paléoclimats du Quaternaire récent dans les Andes centrales, y a-t-il des éléments qui tendent à montrer que ces conditions ont pu être réalisées?

La question des oscillations des températures depuis le Tardiglaciaire dans cette partie des Andes a été abordée par les paléobotanistes et les stratigraphes (Clapperton, 1983). 


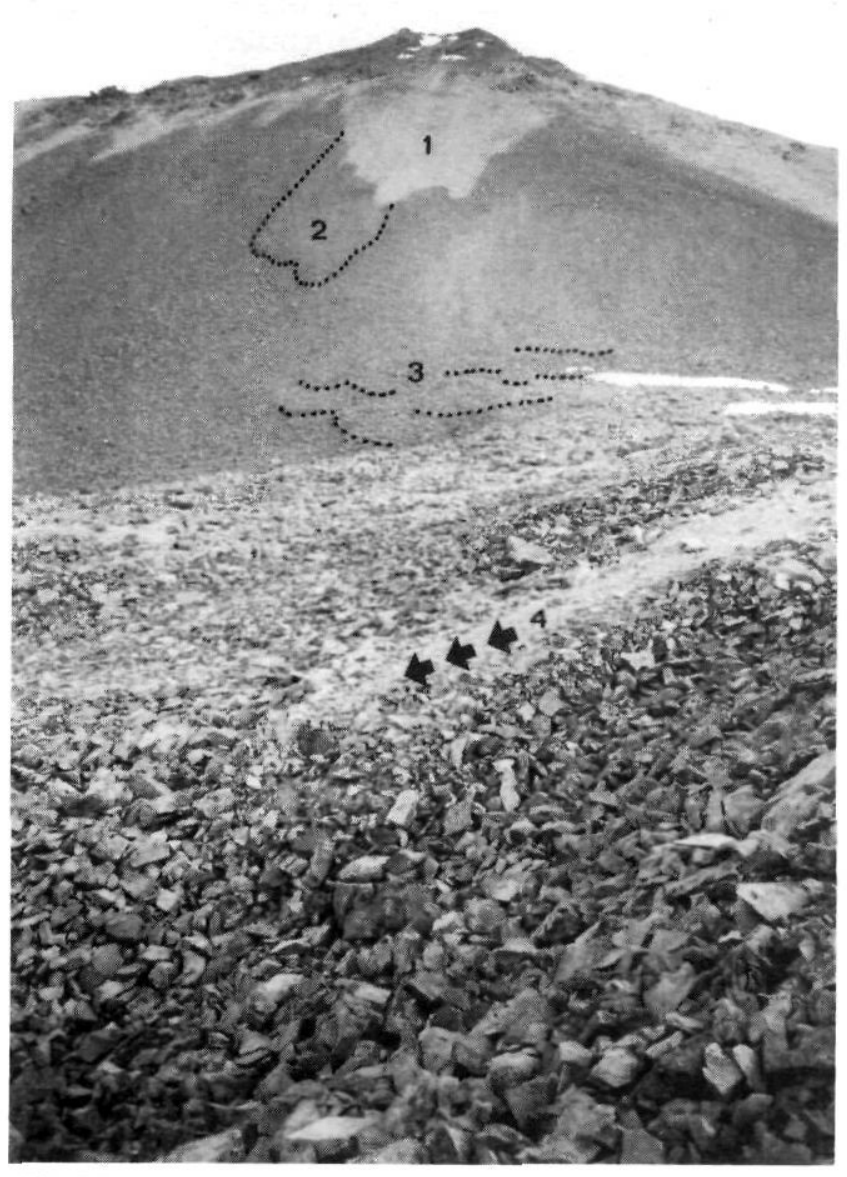

FIGURE 14. L'éboulis $\mathrm{N}$ du Cerro Carrizal W avec la nappe-coulée active (1), la nappe-coulée pierreuse inerte (2) et les bourrelets de la base (3). Au premier plan, coulée à front pierreux (4). Altitude: $5200 \mathrm{~m}$.

The northward talus of the Cerro Carrizal $W$ with the sheet of fine material (1), the sheet with blocky material (2) and the metric ridges at the foot (3). In the foreground, stone-banked lobe. Altitude: $5200 \mathrm{~m}$.

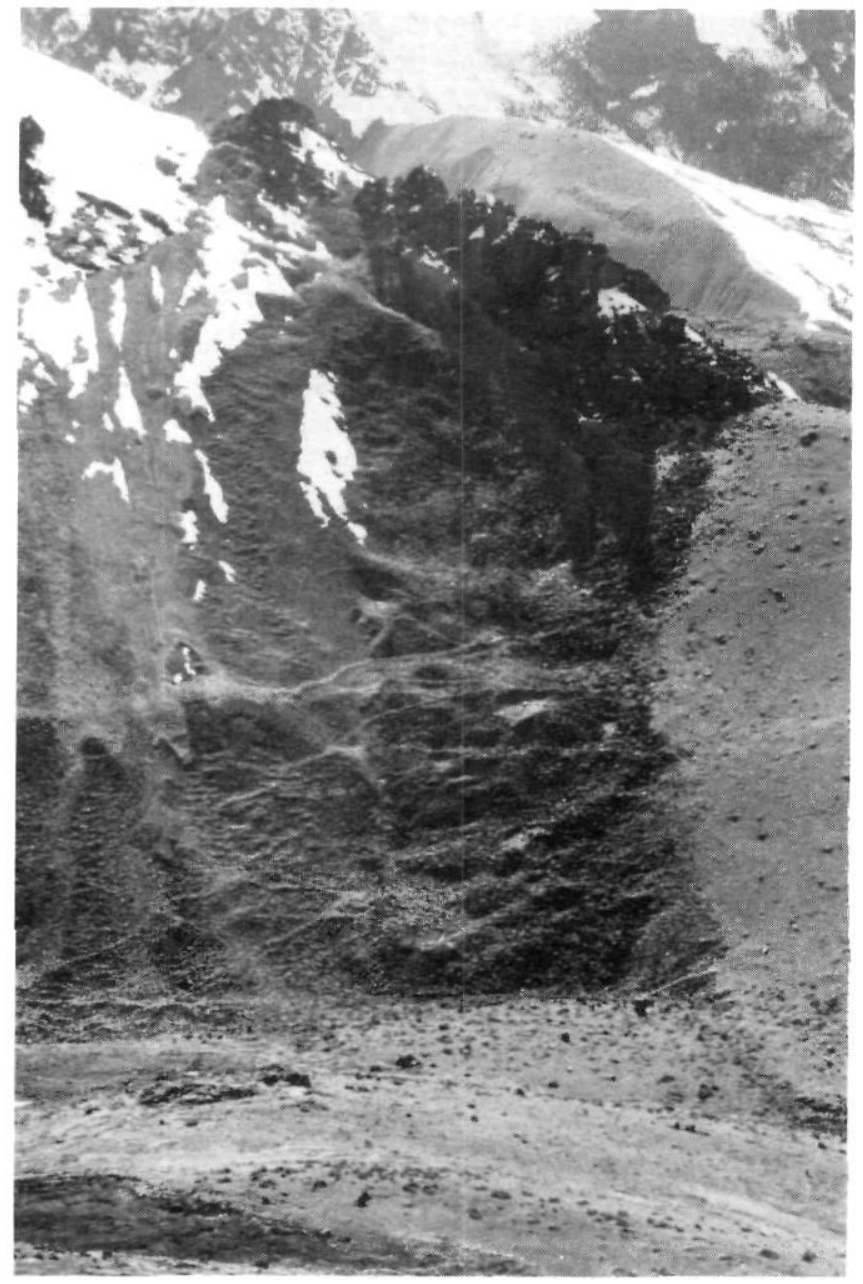

FIGURE 15. Un "éboulis ondulé" sur le versant SW du Cerro Ucrupata à $4800 \mathrm{~m}$. Matériel grossier peu mobile accidenté de bourrelets de solifluxion développés pendant un épisode plus froid. Les nappes fines, de part et d'autre, sont actives.

"Undulating talus" on the SW slope of the Cerro Ucrupata at 4,800 m. Blocky material with solifluction ridges formed during a colder period. Sheets of fine material at the both sides are active.
Depuis la fin du dernier Pléniglaciaire andin, il y a 14000 ans BP (Mercer et Palacios, 1977; Argollo et al., 1987), plusieurs périodes sensiblement plus froides que l'actuelle ont été identifiées en Bolivie ( $\left.16^{\circ} \mathrm{S}\right)$ par la palynologie (Graf, 1981; Ybert, 1984). Certaines se sont produites dans un contexte sec, défavorable à l'extension des glaciers, comme lors de la transition entre le Tardiglaciaire et l'Holocène. Selon Ybert (1984), durant cet épisode, les températures ont dû être de 3 à $6^{\circ} \mathrm{C}$ inférieures à leur niveau actuel, ce qui correspondrait à une dépression des isothermes de $400 \mathrm{~m}$ au minimum et mettrait l'isotherme $0^{\circ} \mathrm{C}$ à $4600 \mathrm{~m}$. Comme on sait par ailleurs (Mercer et Palacios, 1977; Clapperton, 1983; Argollo et al., 1987) que la limite glaciaire n'a que très peu varié depuis 10000 ans, en dehors de l'épisode marqué du Petit Âge glaciaire (500-50 ans BP), un étage périglaciaire plus ample et aux conditions cryonivales accentuées a pu se développer à cette époque. En conséquence, la distribution des précipitations en fonction des saisons et les types de temps devaient être différents avec une saison estivale aux précipitations diminuées, évitant un gonflement du bilan des glaciers et une saison hivernale moins sèche, à forte nébulosité, avec de fréquentes petites chutes de neige. Cette augmentation des précipitations pendant les mois d'hiver ne peut être liée dans une telle zone géographique qu'à de grands déplacements méridiens des masses d'air accompagnés de fronts froids le long de l'axe andin. Ce type de situation n'est pas inconnu aujourd'hui et certains auteurs (Servant et Villaroel, 1979) lui ont attribué une plus grande fréquence au cours du Quaternaire.

On voit donc que l'étude morphodynamique du gel peut apporter des éléments d'un grand intérêt dans le débat sur l'évolution du climat des Andes depuis la période finale du Pléistocène. Il conviendra dans le futur d'affiner les informations que l'étude du gel du sol peut nous fournir, tout en cherchant 
à multiplier dans le même temps les jalons chronologiques sur les paléoformes, notamment celles qui ont été mises en place à des époques où les processus cryonivaux étaient plus actifs et l'étage périglaciaire plus développé.

\section{REMERCIEMENTS}

Nous remercions les lecteurs de la revue, MM. M. Allard, B. Hétu et B. H. Luckman, qui par leurs conseils nous ont aidé à améliorer notre manuscrit.

\section{RÉFÉRENCES}

Argollo, J., Gouze, Ph., Saliège, J. F. et Servant, M., 1987. Fluctuations des glaciers de Bolivie au Quaternaire récent. Géodynamique, 2(2): 103-104.

Benedict, J. B., 1970. Downslope soil movement in a Colorado Alpine region: rates, processes and climatic significance. Arctic and Alpine Research 2(3): 165-226.

Broggi, J. A., 1945. La desglaciación actual de los Andes del Perù. Boletin del Museo de Historia Natural, 34-35: 223-248.

Caine, N., 1963. Movement of low-angle scree slopes in the Lake District, Northern England. Revue de Géomorphologie dynamique, 14: 171-177.

1980. The rainfall intensity-duration control of shallow landslides and debris-flow. Geografiska Annaler, 62A(1-2): 23-27.

Church, M. et Ryder, J. M., 1972. Paraglacial sedimentation: a consideration of fluvial processes conditioned by glaciation. Geological Society of America Bulletin, 83: 3059-3072.

Clapperton, C. M., 1983. The glaciation of the Andes. Quaternary Science Review, 2: 83-155.

Dewolf, Y., 1988. Stratified slope deposits, p. 91-123. In M. J. Clark, édit., Advances in Periglacial Geomorphology. John Willey \& Sons Ltd, Chichester, $480 \mathrm{p}$.

Dollfus, O., 1965. Les Andes Centrales du Pérou et leurs piémonts. Thèse, Université de Paris, Institut français d'études andines, Paris, $404 p$

Fahey, B. D., 1972. An analysis of diurnal freeze-thaw and frost heave cycles in the Indian Peaks region of Colorado Front Range. Arctic and Alpine Research, 5: 269-281.

Francou, B., 1983. Les régimes thermiques et pluviométriques de Pachachaca. Bulletin de l'Institut français d'études andines, Lima, XII, 1-2: 17-53.

1986. Dynamiques périglaciaires et Quaternaire dans les Andes Centrales du Pérou. Rapport scientifique et technique, 2, Centre de géomorphologie du CNRS, Caen, $63 \mathrm{p}$.

1988a. Températures de parois rocheuses et gélifraction dans les Andes Centrales du Pérou: étude à partir de 2 sites. Bulletin du Centre de géomorphologie du CNRS, Caen, 34: 159-180.

1988b. L'éboulisation en haute montagne. Thèse, Université de Paris 7, Éditec, Caen, 696 p.

1988c. Éboulis stratifiés dans les hautes Andes Centrales du Pérou. Zeitschrift für Geomorphologie, 32 (1): 47-76.

Francou, B. et Bourgeat, S., 1988. Dynamiques périglaciaires et Quaternaire dans les Andes Centrales du Pérou II. Rapport scientifique et technique, 4, Centre de géomorphologie du CNRS, Caen, $55 \mathrm{p}$.
Graf, K., 1971. Beiträge zur solifluktion in den Bündner Alpen (Schweiz) und in den Anden Perus und Bolivien. Inaugural Dissertation, Zürich, $152 \mathrm{p}$.

1976. Zür mechanik von frostmusterüngsprozessen in Bolivien und Ecuador. Zeitschrift für Geomorphologie, 20 : 417-447.

1981. Palynological investigations of two Post-Glacial peat bogs near the boundary of Bolivia and Peru. Journal of Biogeography, 8: 353-368.

Hastenrath, S., 1977. Observations on soil frost phenomena in the Peruvian Andes. Zeitschrift für Geomorphologie, 20: 357-362.

Hétu, B., 1986. L'influence du contexte géomorphologique quaternaire sur la dynamique postglaciaire des versants raides de la Gaspésie septentrionale. Thèse, Université de Montréal, $667 \mathrm{p}$.

Meetmeyer, V. et Zippin, J., 1981. Soil moisture and texture control of selected parameters of needle ice growth. Earth Surface Processess and Landforms, 6: 113-125.

Mégard, F., 1973. Étude géologique d'une transversale des Andes au niveau du Pérou central. Thèse, Université des Sciences et des Techniques du Languedoc, Montpellier, $263 \mathrm{p}$.

Mercer, J. H. et Palacios, O., 1977. Radiocarbon dating of the last glaciation in Peru. Geology, 5: 600-604.

Outcalt, S. I., 1971. An algorithm of needle ice growth. Water Resources Research, $7:$ 394-400.

Péguy, Ch.P., 1976. Une nouvelle expression graphique de la variabilité inter-annuelle des climats: les calendriers de probabilités. Bulletin de l'Association des géographes français, 431: 5-16.

Pérez, F. L., 1984. Striated soils in an Andean Paramo of Venezuela: its origin and orientation. Arctic and Alpine Research, 16, 3:277289.

1985. Surficial talus movement in an Andean Paramo of Venezuela. Geografiska Annaler, 67 A 3-4: 221-236.

1986. Talus texture and particle morphology in a North Andean Paramo. Zeitschrift für Geomorphology, 30, 1: 15-34.

1987. Le transport des cailloux par la glace d'extrusion dans les hautes Andes (Vénézuela). Revue de Géomorphologie dynamique, XXXVI (2): 33-51.

Pissart, A., 1973. L'origine des sols polygonaux et striés du Chambeyron (Basses-Alpes). Bulletin de la Société géographique de Liège, 9: 33-53.

Schubert, C., 1973. Striated ground in the Venezuela Andes. Journal of Glaciology, 12: 641-466.

1979. La zona del Páramo: morphología glacial y periglacial de los Andes de Venezuela, p. 11-27. In M. L. Salgado-Labouriau, El medio ambiente páramo. Centro de Estudios Avanzados Ed.

Servant, M. et Villaroel, R., 1979. Le problème paléoclimatique des Andes et de leurs piémonts au Quaternaire. Compte rendu de l'Académie des Sciences, Paris, 288, série D: 665-668.

Schwerdtfeger, W., 1976. Introduction, p. 1-12. In Schwerdtfeger, W., édit., World Survey of Climatology, vol. 12, Climate of Central and South America. New York, Elsevier Scientific.

Statham, I., 1976. A scree slope rockfall model. Earth Surface Processes and Landforms, 2: 43-62.

Tricart, J. et Cailleux, A., 1965. Introduction à la géomorphologie climatique. SÉDES, Paris, $512 \mathrm{p}$.

1967. Le modelé des régions périglaciaires. SÉDES, Paris, $512 \mathrm{p}$. 
Troll, C., 1944. Strukturböden, Solifluktion und Frostklimate der Erde. Geologie Rundchau, 34: 545-694.

1968. The cordilleras of the tropical Americas: aspects of climate, phytogeographical and agrarian ecology, p. 15-56. In Troll, C., Geo-Ecology of the mountainous regions of the tropical Americas. Bonn, Ferd. Dummlers Verlag.

Van Vliet-Lanoë, B., 1988. Le rôle de la glace de ségrégation dans les formations superficielles de l'Europe de l'Ouest: processus et héritages. Thèse, Université de Paris-Sorbonne, Éditec, Caen, $854 \mathrm{p}$.

Van Vliet-Lanoë, B. et Francou, B., 1988. Étude micromorphologique et dynamique comparative de sols striés et autres petites formes fluantes superficielles en milieux arctique, alpin et andin. Bulletin du Centre de géomorphologie du CNRS, Caen, 34: 47-64.

Washburn, A. L., 1979. Geocryology. A survey of periglacial processes and environments. Londres, Edward Arnold, $406 \mathrm{p}$.

Whalley, W. B., 1974. The mechanics of high mountains-low frequency rock failure and its importance in mountainous areas. Geographical Papers, 27, Readind University, $48 p$.

Yaïr, A. et Lavee, H., 1974. Areal contribution to runoff on scree slopes in an extreme arid environment: a simulation rainstorm experiment. Zeitschrift für Geomorphologie, S.B., 21: 202-215.

Ybert, J. P., 1984. Diagramme sporopollinique de la coupe holocène du Rio Chuquiaguillo sur l'Altiplano bolivien. Cahier ORSTOM géologie, XIV (1): 29-34. 